\title{
On the convergence of formal mappings
}

\author{
NORDINE MIR
}

Let $f:(M, p) \rightarrow\left(M^{\prime}, p^{\prime}\right)$ be a formal (holomorphic) nondegenerate map, i.e., with formal holomorphic Jacobian $J_{f}$ not identically vanishing, between two germs of real analytic generic submanifolds in $\mathbb{C}^{n}, n \geq 2, p^{\prime}=f(p)$. Assuming the target manifold to be real algebraic, and the source manifold to be minimal at $p$ in the sense of Tumanov, we prove the convergence of the so-called reflection mapping associated to $f$. From this, we deduce the convergence of such mappings from minimal real analytic generic submanifolds into real algebraic holomorphically nondegenerate ones, as well as related results on partial convergence of such maps. For the proofs, we establish a principle of analyticity for formal CR power series. This principle can be used to reobtain the convergence of formal mappings of real analytic CR manifolds under a standard nondegeneracy condition.

\section{Introduction.}

A formal (holomorphic) mapping $f:\left(\mathbb{C}^{n}, p\right) \rightarrow\left(\mathbb{C}^{n^{\prime}}, p^{\prime}\right),\left(p, p^{\prime}\right) \in \mathbb{C}^{n} \times \mathbb{C}^{n^{\prime}}$, $n, n^{\prime} \geq 1$, is a vector $\left(f_{1}, \ldots, f_{n^{\prime}}\right)$ where each $f_{j} \in \mathbb{C}[[z-p]]$ is a formal holomorphic power series in $z-p$, and $f(p)=p^{\prime}$. In the case $n=n^{\prime}$, a formal mapping $f$ is called nondegenerate if its formal holomorphic Jacobian $J_{f}$ does not vanish identically as a formal power series in $z-p$. An important class of nondegenerate formal maps $f$ consists of those which are invertible, namely those for which $J_{f}(p) \neq 0$. We call such maps formal equivalences or formal invertible maps. If $M, M^{\prime}$ are two smooth real real analytic generic submanifolds in $\mathbb{C}^{n}$ and $\mathbb{C}^{n^{\prime}}$ respectively (through $p, p^{\prime}$ respectively) and of real codimension $c$ and $c^{\prime}$ respectively, we say that a formal mapping $f:\left(\mathbb{C}^{n}, p\right) \rightarrow\left(\mathbb{C}^{n^{\prime}}, p^{\prime}\right)$ sends $M$ into $M^{\prime}$ if

$$
\rho^{\prime}(f(z), \overline{f(z)})=a(z, \bar{z}) \cdot \rho(z, \bar{z})
$$

where $\rho=\left(\rho_{1}, \ldots, \rho_{c}\right)$ and $\rho^{\prime}=\left(\rho_{1}^{\prime}, \ldots, \rho_{c^{\prime}}^{\prime}\right)$ are local real analytic defining functions for $M, M^{\prime}$ respectively and $a(z, \bar{z})$ is a $c^{\prime} \times c$ matrix with entries in $\mathbb{C}[[z-p, \bar{z}-\bar{p}]]$. It is easy to see that such a definition is independent of 
the choice of defining functions for $M$ and $M^{\prime}$. If $f$ is formal mapping as above sending $M$ into $M^{\prime}$, we may also say that $f$ is a formal $C R$ mapping from $M$ into $M^{\prime}$. This is motivated by the fact that, if, in addition, $f$ is convergent near $p$, then $f$ is a real analytic CR mapping from $M$ into $M^{\prime}$.

A natural question which arises is to give necessary and sufficient conditions so that any formal equivalence between real analytic generic submanifolds must be convergent. Chern and Moser [9] gave the first results in this direction by proving the convergence of formal equivalences between Levi nondegenerate real analytic hypersurfaces. Later, Moser and Webster [22] showed the analyticity of formal invertible mappings between certain real analytic surfaces of dimension two in $\mathbb{C}^{2}$, but which are not $\mathrm{CR}$. Other related work was done by Webster [27] and Gong [15]. (We also refer the reader to the bibliography given in [6] for further information.) More recently, Baouendi, Ebenfelt and Rothschild proved the convergence of formal equivalences between minimal finitely nondegenerate real analytic generic submanifolds $[4,5]$, as well as between minimal essentially finite ones ${ }^{1}[6]$ (other situations are also treated in $[5,6]$ ). The conditions of finite nondegeneracy and essential finiteness are closely related to the notion of holomorphic nondegeneracy introduced by Stanton [24]. Let us recall that a connected real analytic generic submanifold is holomorphically nondegenerate if, near any point $p \in M$, there is no non-trivial holomorphic vector field, with holomorphic coefficients, tangent to $M$ near $p$. Such submanifolds have the property to be generically essentially-finite in the sense that, for any such manifold $M$, there always exist a proper real analytic subvariety $S \subset M$ (which may be empty) such that any point $p \in M \backslash S$ is essentially finite. Moreover, it was observed in [4] that the condition of holomorphic nondegeneracy is necessary for the convergence of formal equivalences between real analytic generic submanifolds. Thus, to complete the previous results, one has to treat the case of the non-essentially finite points of such holomorphically nondegenerate submanifolds.

In the one-codimensional case, these non-essentially finite points were recently treated in [21] where, in particular, it was shown that any formal $\mathrm{CR}$ equivalence between minimal holomorphically nondegenerate real analytic hypersurfaces must be convergent. The goal of this paper is to study the higher-codimensional case. Assuming the target manifold to be real algebraic i.e., contained in a real algebraic subvariety of the same dimension, we establish a result which gives a description of the analyticity properties of formal CR nondegenerate maps from minimal real analytic generic sub-

\footnotetext{
${ }^{1}$ See $\S 3$ for precise definitions.
} 
manifolds of $\mathbb{C}^{n}$ into real algebraic ones (Theorem 2.1 below). As in $[20,21]$, we prove that, given a formal map $f:(M, p) \rightarrow\left(M^{\prime}, p^{\prime}\right)$ with $J_{f} \not \equiv 0$, if $M$ is minimal at $p$, then the so-called associated reflection mapping (cf. [17]) must be convergent. As we shall see $(c f$. $\S 8)$, such a result can be seen as a result of partial convergence for formal CR nondegenerate maps. This allows one also to deduce the convergence of such maps from real analytic minimal generic submanifolds onto real algebraic holomorphically nondegenerate ones (Theorem 2.2 below). We should point out that our arguments give also a quite simple proof of such a fact (see Proposition 7.2). In fact, the algebraicity of the target manifold allows us to use certain tools from basic field theory that we introduced in our previous works $[19,20]$.

\section{Statement of main results.}

Let $\left(M^{\prime}, p^{\prime}\right) \subset \mathbb{C}^{n}$ be a germ at $p^{\prime}$ of a real algebraic generic submanifold of $\mathrm{CR}$ dimension $N$ and of real codimension $c, N+c=n$. This means that there exists $\rho^{\prime}(\zeta, \bar{\zeta})=\left(\rho_{1}^{\prime}(\zeta, \bar{\zeta}), \ldots, \rho_{c}^{\prime}(\zeta, \bar{\zeta})\right) c$ real polynomials such that near $p^{\prime}$

$$
M^{\prime}=\left\{\zeta \in\left(\mathbb{C}^{n}, p^{\prime}\right): \rho^{\prime}(\zeta, \bar{\zeta})=0\right\},
$$

with $\bar{\partial} \rho_{1}^{\prime} \wedge \ldots \wedge \bar{\partial} \rho_{c}^{\prime} \neq 0$, on $M^{\prime}$. We shall assume, without loss of generality, that $p^{\prime}$ is the origin. Then, for any point $\omega$ close to 0 , one defines its associated Segre variety to be the $n-c$ dimensional complex submanifold

$$
Q_{\omega}^{\prime}=\left\{\zeta \in\left(\mathbb{C}^{n}, 0\right): \rho^{\prime}(\zeta, \bar{\omega})=0\right\} .
$$

Moreover, since $M^{\prime}$ is generic, renumbering the coordinates if necessary, and after applying the implicit function theorem, one can assume that any Segre variety can be described as a graph of the form

$$
Q_{\omega}^{\prime}=\left\{\zeta \in\left(\mathbb{C}^{n}, 0\right): \bar{\zeta}^{*}=\bar{\Phi}^{\prime}\left(\omega, \bar{\zeta}^{\prime}\right)\right\}, \quad \zeta=\left(\zeta^{\prime}, \zeta^{*}\right) \in \mathbb{C}^{N} \times \mathbb{C}^{c}
$$

$\bar{\Phi}^{\prime}=\left(\bar{\Phi}_{1}^{\prime}, \ldots, \bar{\Phi}_{c}^{\prime}\right)$ denoting a convergent power series mapping near $0 \in$ $\mathbb{C}^{n+N}$, with $\bar{\Phi}^{\prime}(0)=0$. Our main result is the following.

Theorem 2.1. Let $f:(M, 0) \rightarrow\left(M^{\prime}, 0\right)$ be a formal nondegenerate $C R$ map between two germs at 0 of real analytic generic submanifolds in $\mathbb{C}^{n}$ of the same $C R$ dimension. Assume that $M$ is minimal at $0 \in M$ and that $M^{\prime}$ is real algebraic. Then, the formal holomorphic map

$$
\mathbb{C}^{n} \times \mathbb{C}^{N} \ni(z, \theta) \mapsto \bar{\Phi}^{\prime}(f(z), \theta) \in \mathbb{C}^{c}
$$

is convergent. 
Such a result was established in [21] in the one-codimensional case (for unbranched mappings) without assuming that the target manifold $M^{\prime}$ is real algebraic. As in [21], Theorem 2.1 allows us to derive the following convergence result.

Theorem 2.2. Let $f:(M, 0) \rightarrow\left(M^{\prime}, 0\right)$ be a formal nondegenerate $C R$ map between two germs at 0 of real analytic generic submanifolds in $\mathbb{C}^{n}$ of the same $C R$ dimension. Assume that $M$ is minimal at $0 \in M$ and that $M^{\prime}$ is real algebraic and holomorphically nondegenerate. Then $f$ is convergent.

As mentioned in the introduction, Theorem 2.2, for unbranched maps, follows from [21] in the hypersurface case, but in the higher codimensional case the result is new and was not previously known even in the case where $M$ and $M^{\prime}$ are both algebraic. Another application of Theorem 2.1 is given in $\S 8$ and deals with partial convergence of formal CR nondegenerate maps. For this, we refer the reader to Theorem 8.1 and Corollary 8.3.

Our approach for proving Theorem 2.1 is essentially based on two steps. The first step is a formulation of the reflection principle via the jet method and follows [20]. The general idea is to show that, under the assumptions of Theorem 2.1, the composition of any component of the Segre variety map of $M^{\prime}$ (as defined in $\S 4$ ) with the map $f$ satisfy certain polynomial equations restricted on $M$, and, more precisely, is algebraic over a certain field of formal power series. The second step consists in proving that a formal CR power series (i.e., a formal holomorphic power series) which satisfies such a polynomial identity, is necessarily convergent (Theorem 5.1). This is based on the theory of Segre sets by Baouendi, Ebenfelt and Rothschild [3], and on some of their techniques of propagation. One should, however, point out several differences with the methods of $[3,6]$ (see especially Proposition 5.5).

The paper is organized as follows. $\S 3$ contains some background material for the reader's convenience. In $\S 4$, we use some ideas from [20] to prove our reflection identities. $\S 5$ is devoted to the proof of a principle of analyticity for formal CR functions. Such a result (Theorem 5.1) seems to us interesting in itself. In $\S 6$, we prove the main results of the paper. In $\S 7$, we formulate some remarks concerning Theorem 2.2 which show that, under the assumptions of that Theorem, the convergence of formal nondegenerate maps can be derived in a quite simple manner. In $\S 8$, we apply Theorem 2.1 to the study of partial convergence for formal CR maps. Finally, in $\S 9$, we apply the principle proved in $\S 5$ to establish the convergence of formal mappings between real analytic CR manifolds under a standard nondegeneracy condition. 
Acknowledgements. This work has been completed while I was invited by the department of Mathematics of the university of Wuppertal, Germany, during the period May-July 1999. I would like to thank Prof. K. Diederich, C. Eppel and Prof. G. Herbort for arranging my visit. I would like also to thank Prof. K. Diederich for interesting conversations. I am indebted to Prof. M. Derridj for his precious help, all his encouragements and for having spent much of his time thinking of my numerous questions. Finally, I wish also to thank the referees of this paper for many valuable comments and helpful suggestions.

\section{Preliminaries, notations and definitions.}

\subsection{Finite nondegeneracy, essential finiteness and holomorphic nondegeneracy of real analytic generic submanifolds.}

Let $M$ be a real analytic generic submanifold through $p \in \mathbb{C}^{n}$, of $\mathrm{CR}$ dimension $N$ and of real codimension $c, N+c=n$. We shall always assume that $c, N \geq 1$, and, for convenience, that the reference point $p$ is the origin. Let $\rho=\left(\rho_{1}, \ldots, \rho_{c}\right)$ be a set of real analytic defining functions for $M$ near 0 , i.e.,

$$
M=\left\{z \in\left(\mathbb{C}^{n}, 0\right): \rho(z, \bar{z})=0\right\},
$$

with $\bar{\partial} \rho_{1} \wedge \ldots \wedge \bar{\partial} \rho_{c} \neq 0$, on $M$. The complexification $\mathcal{M}$ of $M$ is the $2 n-c$-dimensional complex submanifold of $\mathbb{C}^{2 n}$ given by

$$
\mathcal{M}=\left\{(z, w) \in\left(\mathbb{C}^{2 n}, 0\right): \rho(z, w)=0\right\} .
$$

We shall assume, without loss of generality, that the matrix $\partial \rho / \partial z^{*}$ is not singular at the origin, $z=\left(z^{\prime}, z^{*}\right) \in \mathbb{C}^{N} \times \mathbb{C}^{c}$. In this case, we define the following vector fields tangent to $\mathcal{M}$,

$$
\mathcal{L}_{j}=\frac{\partial}{\partial w_{j}}-\rho_{w_{j}}(z, w)\left[\frac{\partial \rho}{\partial w^{*}}(z, w)\right]^{-1} \frac{\partial}{\partial w^{*}}, j=1, \ldots, N,
$$

which are the complexifications of the $(0,1)$ vector fields tangent to $M$. Let us also recall that the invariant Segre varieties attached to $M$ are defined by

$$
Q_{w}=\left\{z \in\left(\mathbb{C}^{n}, 0\right): \rho(z, \bar{w})=0\right\},
$$

for $w$ close to 0 . A fundamental map which arises in the mapping problems is the so-called Segre variety map $\lambda: w \mapsto Q_{w}(c f .[14,12,13])$. A real analytic 
generic submanifold $M$ is called finitely nondegenerate at $p=0 \in M$ if

$$
\operatorname{Span}_{\mathbb{C}}\left\{\mathcal{L}^{\alpha} \rho_{j, z}(p, \bar{p}): \alpha \in \mathbb{N}^{N}, 1 \leq j \leq d\right\}=\mathbb{C}^{n}
$$

Here, for $1 \leq j \leq d, \rho_{j, z}$ denotes the complex gradient of $\rho_{j}$ with respect to $z$. In this case, one can show that the Segre variety map $\lambda$ is actually one-to-one near $p=0$. More generally, $M$ is called essentially finite at $0 \in M$ if the Segre variety map $\lambda$ is finite-to-one near $0[14,7]$. The interest of such conditions lies in the fact that, given a holomorphically nondegenerate generic real analytic submanifold $M$ (as defined in the introduction), the set of finitely nondegenerate or essentially finite points is always, at least, dense in $M$ (see [3]). Furthermore, the set of points $w \in M$ such that $\lambda^{-1}\left(Q_{w}\right)$ is positive-dimensional forms a proper (possibly empty) real analytic subvariety $S \subset M$, provided that the submanifold $M$ is holomorphically nondegenerate. This set of points is precisely the set of non-essentially finite points of $M$.

\subsection{Minimality condition in terms of Segre sets.}

Another nondegeneracy condition which will be used in this paper is the minimality condition introduced by Tumanov [25]. Let us recall that a real analytic generic submanifold $M$ is said to be minimal at $p \in M$ (or of finite type in the sense of Kohn and Bloom-Graham) if there is no proper CR submanifold contained in $M$ through $p$, and with the same CauchyRiemann dimension. In order to give a characterization of minimality for real analytic CR manifolds, Baouendi, Ebenfelt and Rothschild introduced the so-called Segre sets in [3]. These sets will play an important role in our proofs. They are defined as follows. Define the first Segre set $N_{1}(p)$ attached to $M$ at $p \in M$ to be the classical Segre variety $Q_{p}$. Inductively, for $k \in \mathbb{N}$, define

$$
N_{k+1}(p)=\bigcup_{q \in N_{k}(p)} Q_{q}
$$

Recall that the sets $N_{j}(p)$ are in general not analytic for $j>1$. If $M$ is given by (3.1) near $p=0$, as in $\S 3.1$, by the implicit function theorem, one can choose coordinates $z=\left(z^{\prime}, z^{*}\right) \in \mathbb{C}^{N} \times \mathbb{C}^{c}$ so that any Segre variety can be described as a graph as follows

$$
Q_{w}=\left\{z \in\left(\mathbb{C}^{n}, 0\right): z^{*}=\Phi\left(\bar{w}, z^{\prime}\right)\right\}
$$


$\Phi=\left(\Phi_{1}, \ldots, \Phi_{c}\right)$ being a $\mathbb{C}^{c}$-valued holomorphic map near $0 \in \mathbb{C}^{N+n}$, $\Phi(0)=0$. The reality of $M$ also implies that

$$
\Phi\left(w^{\prime}, \bar{\Phi}\left(z^{\prime}, z^{*}, w^{\prime}\right), z^{\prime}\right) \equiv z^{*},\left(z^{\prime}, z^{*}, w^{\prime}\right) \in \mathbb{C}^{N} \times \mathbb{C}^{c} \times \mathbb{C}^{N} .
$$

(Here and in what follows, for any formal power series $g(x) \in \mathbb{C}[[x]], x=$ $\left(x_{1}, \ldots, x_{k}\right), \bar{g}(x)$ is the formal power series obtained by taking the complex conjugates of the coefficients of $g$.) The coordinates are said to be normal for $M$ if, moreover, the condition $\Phi(z, 0) \equiv z^{*}$ holds. It is well known ( $c f$. $[9,7])$ that given a real analytic generic submanifold $M$, one can always find such coordinates. With these notations (and without assuming that the $z$-coordinates are normal for $M$ ), the Segre sets can be parametrized by the following mappings $\left(v_{k}\right)_{k \in \mathbb{N}}$, called the Segre sets mappings. First set $v_{0}:=0 \in \mathbb{C}^{n}$. Inductively, $N_{2 k+1}, k \geq 0$, can be parametrized by the map

$$
\begin{aligned}
\left(\mathbb{C}^{(2 k+1) N}, 0\right) \ni & \left(t_{1}, t_{2}, \ldots, t_{2 k+1}\right) \mapsto \\
v_{2 k+1}\left(t_{1}, \ldots, t_{2 k+1}\right) & :=\left(t_{1}, \Phi\left(\bar{v}_{2 k}\left(t_{2}, \ldots, t_{2 k+1}\right), t_{1}\right)\right)
\end{aligned}
$$

and $N_{2 k}$ by

$$
\begin{aligned}
\left(\mathbb{C}^{2 k N}, 0\right) \ni\left(t_{1}, t_{2}, \ldots, t_{2 k}\right) \mapsto \\
v_{2 k}\left(t_{1}, \ldots, t_{2 k}\right):=\left(t_{1}, \Phi\left(\bar{v}_{2 k-1}\left(t_{2}, \ldots, t_{2 k}\right), t_{1}\right)\right) .
\end{aligned}
$$

Notice that, for any nonnegative integer $b$,

$$
\left(v_{b+1}\left(t_{1}, \ldots, t_{b+1}\right), \bar{v}_{b}\left(t_{2}, \ldots, t_{b+1}\right)\right) \in \mathcal{M} .
$$

We can now state a useful characterization of minimality which is contained in $[2]$.

Theorem 3.1 ([2]). If $M$ is minimal at 0 , there exists $d_{0} \in \mathbb{N}$ large enough such that, in any neighborhood $\mathcal{O}$ of $0 \in \mathbb{C}^{d_{0} N}$, there exists $\left(t_{1}^{0}, \ldots, t_{d_{0}}^{0}\right) \in \mathcal{O}$, such that $v_{d_{0}}\left(t_{1}^{0}, \ldots, t_{d_{0}}^{0}\right)=0$ and such that $v_{d_{0}}$ is submersive at $\left(t_{1}^{0}, \ldots, t_{d_{0}}^{0}\right)$.

\section{Formal nondegenerate CR maps with values in real algebraic CR manifolds.}

\subsection{Real algebraic CR manifolds and field extensions.}

In this section, we collect and recall some facts from $[18,19,20]$ which will be used in the proof of Theorem 2.1 . 
As in $\S 2$, let $\left(M^{\prime}, p^{\prime}\right)$ be a germ through $p^{\prime}=0 \in \mathbb{C}^{n}$ of a real algebraic generic submanifold of $\mathrm{CR}$ dimension $N$ and of real codimension $c$. Following the notations of that section, let $\rho^{\prime}=\left(\rho_{1}^{\prime}, \ldots, \rho_{c}^{\prime}\right)$ be a set of defining real polynomials for $M^{\prime}$ near 0 . Thus,

$$
M^{\prime}=\left\{\zeta \in\left(\mathbb{C}^{n}, 0\right): \rho^{\prime}(\zeta, \bar{\zeta})=0\right\}
$$

with $\bar{\partial} \rho_{1}^{\prime} \wedge \ldots \wedge \bar{\partial} \rho_{c}^{\prime} \neq 0$, on $M^{\prime}$. We can assume that the coordinates $\zeta$ at the target space are chosen so that if $\zeta=\left(\zeta^{\prime}, \zeta^{*}\right) \in \mathbb{C}^{N} \times \mathbb{C}^{c}$, the matrix $\partial \rho^{\prime} / \partial \zeta^{*}$ is not singular at the origin. This allows one to represent $M^{\prime}$ as follows

$$
M^{\prime}=\left\{\zeta \in\left(\mathbb{C}^{n}, 0\right): \bar{\zeta}^{*}=\bar{\Phi}^{\prime}\left(\zeta, \bar{\zeta}^{\prime}\right)\right\}
$$

$\bar{\Phi}^{\prime}=\left(\bar{\Phi}_{1}^{\prime}, \ldots, \bar{\Phi}_{c}^{\prime}\right)$ being a $\mathbb{C}^{c}$-valued holomorphic algebraic map near $0 \in$ $\mathbb{C}^{N+n}$ with $\bar{\Phi}^{\prime}(0)=0$. (We recall here that a holomorphic function in $k$ variables near 0 is called algebraic if it is algebraic over the quotient field of the polynomials in $k$ indeterminates.) Write, for $\nu=1, \ldots, c$, the expansion

$$
\bar{\Phi}_{\nu}^{\prime}(\omega, \theta)=\sum_{\beta \in \mathbb{N}^{N}} q_{\beta, \nu}(\omega) \theta^{\beta} .
$$

Here, $\omega \in \mathbb{C}^{n}$ and $\theta \in \mathbb{C}^{N}$. We also write

$$
\bar{\Phi}_{\theta^{\alpha}}^{\prime}(\omega, \theta)=\left(\bar{\Phi}_{\theta^{\alpha}, 1}^{\prime}(\omega, \theta), \ldots, \bar{\Phi}_{\theta^{\alpha}, c}^{\prime}(\omega, \theta)\right)=\left(\partial_{\theta}^{\alpha} \bar{\Phi}_{1}^{\prime}(\omega, \theta), \ldots, \partial_{\theta}^{\alpha} \bar{\Phi}_{c}^{\prime}(\omega, \theta)\right) .
$$

With these notations, the Segre variety map $\lambda^{\prime}:\left(\mathbb{C}^{n}, 0\right) \ni \omega \mapsto Q_{\omega}^{\prime}$ associated to $M^{\prime}$ can be identified with the holomorphic map

$$
\left(\mathbb{C}^{n}, 0\right) \ni \omega \mapsto\left(q_{\beta, \nu}(\omega)\right)_{\substack{\beta \in \mathbb{N}^{N} \\ 1 \leq \nu \leq c}} .
$$

Here, the Segre variety $Q_{\omega}^{\prime}$, for $\omega$ close to 0 , is defined by (2.1). The family of holomorphic algebraic functions defined by (4.5) will be denoted $\mathcal{C}$. For $k \in \mathbb{N}^{*}$, let $\mathcal{F}_{k}$ be the quotient field of the germs at $0 \in \mathbb{C}^{k}$ of algebraic functions in $\mathbb{C}^{k}$. For any positive integer $l \in \mathbb{N}$, we define $\mathcal{P}_{l}$ to be the smallest field contained in $\mathcal{F}_{N+n}$ and containing $\mathbb{C}$ and the family $\left(\theta, \bar{\Phi}_{\theta^{\beta}, j}^{\prime}(\omega, \theta)\right)_{j=1, \ldots, c,|\beta| \leq l}$. We then define $\mathcal{P} \subset \mathcal{F}_{N+n}$ to be the set

$$
\mathcal{P}=\cup_{l \in \mathbb{N}} \mathcal{P}_{l} .
$$

One can easily check that $\mathcal{P}$ is also a subfield of $\mathcal{F}_{N+n}$, since, for any $l$, $\mathcal{P}_{l} \subset \mathcal{P}_{l+1}$. By definition, an element $b=b(\omega, \theta) \in \mathcal{F}_{N+n}$ belongs to $\mathcal{P}$ if 
there exists a positive integer $l$ and two holomorphic polynomials $Q_{1}$ and $Q_{2}$ such that $Q_{2}\left(\left(\bar{\Phi}_{\theta^{\beta}, j}^{\prime}(\omega, \theta)\right)_{j \leq c,|\beta| \leq l}, \theta\right) \not \equiv 0$ in $\mathcal{F}_{N+n}$ and such that

$$
b=b(\omega, \theta)=\frac{Q_{1}\left(\left(\bar{\Phi}_{\theta^{\beta}, j}^{\prime}(\omega, \theta)\right)_{j \leq c,|\beta| \leq l}, \theta\right)}{Q_{2}\left(\left(\bar{\Phi}_{\theta^{\beta}, j}^{\prime}(\omega, \theta)\right)_{j \leq c,|\beta| \leq l}, \theta\right)} .
$$

We need to state the following proposition, established in [20] (Proposition 1) in the hypersurface case, but which follows with the same proof in the higher codimensional case.

Proposition 4.1. Let $M^{\prime}$ be a real algebraic generic submanifold of $C R$ dimension $N$ through the origin in $\mathbb{C}^{n}$. Assume that $M^{\prime}$ is given near 0 by (4.2). Let $\mathcal{C}$ be the family of algebraic holomorphic functions (in $n$ variables) defined by (4.5) and $\mathcal{P}$ be the field of algebraic holomorphic functions (in $N+n$ variables) defined by (4.6). Then the following holds. The family $\mathcal{C}$ is contained in the algebraic closure of $\mathcal{P}$, and hence, the algebraic closure of $\mathcal{C}$ is contained in the algebraic closure of $\mathcal{P}$.

Remark 1. An inspection of the proof of Proposition 1 from [20] shows that there exists $l_{0}$, which depends only on $M^{\prime}$ such that $\mathcal{C}$ is contained in the algebraic closure of $\mathcal{P}_{l_{0}}$. Moreover, if $M^{\prime}$ is holomorphically nondegenerate, $l_{0}$ is nothing else than the so-called Levi-type of $M^{\prime}$ (see [3]). Indeed, we define $l_{0}$ as follows. Consider, for any positive integer $l$, the $\operatorname{map} \psi_{l}:\left(\mathbb{C}^{N+n}, 0\right) \ni(\omega, \theta) \mapsto\left(\theta,\left(\bar{\Phi}_{\theta^{\beta}, j}^{\prime}(\omega, \theta)\right)_{j \leq c,|\beta| \leq l}\right)$ and denote by $r_{l}$ the generic rank of such a map. Finally, put $r\left(M^{\prime}\right)=\max _{l \in \mathbb{N}} r_{l}$. Then, $l_{0}=\inf \left\{l \in \mathbb{N}: r_{l}=r\left(M^{\prime}\right)\right\}$. When $M^{\prime}$ is holomorphically nondegenerate, then it is well-known ( $c f$. [3]) that, in that case, the integer $r\left(M^{\prime}\right)$ equals $N+n$ and, by definition, $l_{0}$ is the Levi-type of $M^{\prime}$.

We recall also the following criterion of holomorphic nondegeneracy from $[18,19]$.

Theorem 4.2. Let $M^{\prime}$ be a real algebraic generic submanifold through the origin in $\mathbb{C}^{n}$. Assume also that $M^{\prime}$ is given near 0 by (4.2). Let $\mathcal{C}$ be the family of algebraic holomorphic functions (in $n$ variables) defined by (4.5) and $\mathcal{P}$ be the field of algebraic holomorphic functions (in $N+n$ variables) defined by (4.6). Then, the following conditions are equivalent:

(i) $M^{\prime}$ is holomorphically nondegenerate (at 0 ); 
(ii) The algebraic closure of the field $\mathcal{P}$ is $\mathcal{F}_{N+n}$;

(iii) The algebraic closure of the field generated by $\mathcal{C}$ is $\mathcal{F}_{n}$.

\subsection{Jets and the reflection principle.}

In this section, we assume that we are in the following setting. Let $f:(M, p) \rightarrow\left(M^{\prime}, p^{\prime}\right)$ be a formal CR map between two real analytic generic submanifolds in $\mathbb{C}^{n}$, with the same CR dimension $N$ and same real codimension $c$. We assume that $f$ is a nondegenerate map, i.e., that its formal holomorphic Jacobian $J_{f}$ is not identically vanishing. We also assume that $M^{\prime}$ is a real algebraic generic submanifold and, without loss of generality, that $p$ and $p^{\prime}$ are the origin. We use the notations introduced in $\S 3$ for $M$, and those introduced in $\S 4.1$ for $M^{\prime}$. The goal of this section is to prove the following proposition.

Proposition 4.3. Let $M \subset \mathbb{C}^{n}$ be a real analytic generic submanifold through the origin and $M^{\prime} \subset \mathbb{C}^{n}$ be a real algebraic generic submanifold through the origin with the same $C R$ dimension. Assume that $M^{\prime}$ is given near 0 by (4.2). Let $\mathcal{C}$ be the family of algebraic functions (in $n$ variables) associated to $M^{\prime}$ defined by (4.5). Let $\chi \in \mathcal{C}$ and $f: M \rightarrow M^{\prime}$ be a formal $C R$ map between $M$ and $M^{\prime}$ with $J_{f} \not \equiv 0$. Then there exists $l_{0} \in \mathbb{N}^{*}$ (depending only on $M^{\prime}$ ), a positive integer $k_{0}$ (depending only on $M^{\prime}$ and $\chi)$ and a family of convergent power series $\delta_{i}=\delta_{i}\left(\left(\Lambda_{\gamma}\right)_{|\gamma| \leq l_{0}}, z, w\right) \in$ $\mathbb{C}\left\{\left(\Lambda_{\gamma}-\partial^{\gamma} \bar{f}(0)\right)_{|\gamma| \leq l_{0}}, z, w\right\}, i=0, \ldots, k_{0}$, such that the formal power series identity

$$
\sum_{i=0}^{k_{0}} \delta_{i}\left(\left(\partial^{\gamma} \bar{f}(w)\right)_{|\gamma| \leq l_{0}}, z, w\right)((\chi \circ f)(z))^{i}=0
$$

holds for $(z, w) \in \mathcal{M}$ such that $\delta_{k_{0}}\left(\left(\partial^{\gamma} \bar{f}(w)\right)_{|\gamma| \leq l_{0}}, z, w\right) \not \equiv 0$ in $\mathcal{M}$. Here, $\mathcal{M}$ is the complexification of $M$ as defined by (3.2).

To prove Proposition 4.3, we will use an approach which is contained in [20]. We shall first state several preliminary results needed for its proof.

Recall first that the coordinates at the target space are denoted by $\zeta$. We write

$$
f=\left(f^{\prime}, f^{*}\right)=\left(f_{1}^{\prime}, \ldots, f_{N}^{\prime}, f^{*}\right)
$$

in the $\zeta=\left(\zeta^{\prime}, \zeta^{*}\right) \in \mathbb{C}^{N} \times \mathbb{C}^{c}$ coordinates. Since $f$ maps formally $M$ into $M^{\prime}$, there exists $a(z, \bar{z})$ a $c \times c$ matrix with coefficients in $\mathbb{C}[[z, \bar{z}]]$ such that 
the following formal vectorial identity

$$
\overline{f^{*}(z)}-\bar{\Phi}^{\prime}\left(f(z), \overline{f^{\prime}(z)}\right)=a(z, \bar{z}) \cdot \rho(z, \bar{z})
$$

holds. Equivalently this gives

$$
\bar{f}^{*}(w)-\bar{\Phi}^{\prime}\left(f(z), \bar{f}^{\prime}(w)\right)=a(z, w) \cdot \rho(z, w), \text { in } \mathbb{C}[[z, w]] .
$$

Define

$$
D(z, w)=\operatorname{det}\left(\mathcal{L}_{j} \bar{f}_{i}^{\prime}(w)\right)_{1 \leq i, j \leq N} \in \mathbb{C}[[z, w]]
$$

Here, $\mathcal{L}_{j}$, for $j=1, \ldots, N$, is the vector field defined by (3.3). By applying the vector fields $\mathcal{L}_{j}, j=1, \ldots, N$, to (4.9) and Cramer's rule, one obtains the following known lemma (cf. [5, 12]).

Lemma 4.4. Let $f:(M, 0) \rightarrow\left(M^{\prime}, 0\right)$ be a formal $C R$ mapping as in Proposition 4.3. With the notations introduced in (4.10) and (4.4), the following holds. For any multi-index $\alpha \in \mathbb{N}^{N}$, one has the following c-dimensional formal identity

$$
D^{2|\alpha|-1}(z, w) \bar{\Phi}_{\theta^{\alpha}}^{\prime}\left(f(z), \bar{f}^{\prime}(w)\right)=V_{\alpha}\left(\left(\partial^{\beta} \bar{f}(w)\right)_{|\beta| \leq|\alpha|}, z, w\right)
$$

for $(z, w) \in \mathcal{M}$. Here, $V_{\alpha}=\left(V_{\alpha}^{1}, \ldots, V_{\alpha}^{c}\right) \in\left(\mathbb{C}\left\{\left(\Lambda_{\beta}-\partial^{\beta} \bar{f}(0)\right)_{|\beta| \leq|\alpha|}, z, w\right\}\right)^{c}$.

The following lemma contains two known and easy facts.

Lemma 4.5. Let $f:(M, 0) \rightarrow\left(M^{\prime}, 0\right)$ be a formal $C R$ mapping as in Proposition 4.3. Let $D$ be as in (4.10). Then the following holds.

(i) There exists a convergent power series

$$
U=U\left(z, w,\left(\Lambda_{\beta}\right)_{|\beta|=1}\right) \in \mathbb{C}\left\{z, w,\left(\Lambda_{\beta}-\partial^{\beta} \bar{f}(0)\right)_{|\beta|=1}\right\}
$$

such that

$$
D(z, w)=U\left(z, w,\left(\partial^{\beta} \bar{f}(w)\right)_{|\beta|=1}\right) .
$$

(ii) $D(z, w) \not \equiv 0$ for $(z, w) \in \mathcal{M}$.

Proof of Proposition 4.3. Let $\mathcal{P}$ be the subfield of $\mathcal{F}_{N+n}$ defined by (4.6). We also recall that for the positive integer $l_{0}$ mentioned in Remark $1, \mathcal{P}_{l_{0}}$ is the smallest field contained in $\mathcal{F}_{N+n}$ and containing $\mathbb{C}$ and the family 
$\left.\left(\theta, \bar{\Phi}_{\theta^{\beta}, j}^{\prime}(\omega, \theta)\right)_{1 \leq j \leq c,|\beta| \leq l_{0}}\right)$. Let $\chi \in \mathcal{C}$. Since, by Proposition 4.1, $\chi$ is algebraic over $\mathcal{P}$ and, according to Remark 1 , also over $\mathcal{P}_{l_{0}}$, we obtain the existence of a positive integer $k_{0}$ and a family $\left(b_{j}(\omega, \theta)\right)_{0 \leq j \leq k_{0}-1} \subset \mathcal{P}_{l_{0}}$ such that the following identity

$$
(\chi(\omega))^{k_{0}}+\sum_{j=0}^{k_{0}-1} b_{j}(\omega, \theta)(\chi(\zeta))^{j} \equiv 0
$$

holds in the field $\mathcal{F}_{N+n}$. By definition, for $j=0, \ldots, k_{0}-1$, there exist holomorphic polynomials $Q_{1, j}, Q_{2, j}$ such that

$$
Q_{2, j}\left(\left(\bar{\Phi}_{\theta^{\beta}, j}^{\prime}(\omega, \theta)\right)_{j \leq c,|\beta| \leq l_{0}}, \theta\right) \not \equiv 0
$$

and such that

$$
b_{j}(\omega, \theta)=\frac{Q_{1, j}\left(\left(\bar{\Phi}_{\theta^{\beta}, j}^{\prime}(\omega, \theta)\right)_{j \leq c,|\beta| \leq l_{0}}, \theta\right)}{Q_{2, j}\left(\left(\bar{\Phi}_{\theta^{\beta}, j}^{\prime}(\omega, \theta)\right)_{j \leq c,|\beta| \leq l_{0}}, \theta\right)} .
$$

Now, one sees that (4.11), (4.12) and (4.13) imply that there exist holomorphic polynomials $s_{j}, j=0, \ldots, k_{0}$, such that, in some neighborhood of $0 \in \mathbb{C}^{2 n-c}$, the following identity

$$
\sum_{i=0}^{k_{0}} s_{i}\left(\left(\bar{\Phi}_{\theta^{\alpha}, \mu}^{\prime}(\omega, \theta)\right)_{\mu \leq c,|\alpha| \leq l_{0}}, \theta\right)(\chi(\omega))^{i} \equiv 0
$$

holds, with the additional non-degeneracy condition

$$
s_{k_{0}}\left(\left(\bar{\Phi}_{\theta^{\alpha}, \mu}^{\prime}(\omega, \theta)\right)_{\mu \leq c,|\alpha| \leq l_{0}}, \theta\right) \not \equiv 0 .
$$

Note that $k_{0}$ and the family $\left(s_{i}\right)_{i \leq k_{0}}$ depend only on $\chi$ and $M^{\prime}$. Putting, for $(z, w) \in \mathcal{M}, \omega=f(z)$ and $\theta=\bar{f}^{\prime}(w)$ in (4.14), one obtains the following formal identity (cf. [20])

$$
\sum_{i=0}^{k_{0}} s_{i}\left(\left(\bar{\Phi}_{\theta^{\alpha}, \mu}^{\prime}\left(f(z), \bar{f}^{\prime}(w)\right)\right)_{\mu \leq c,|\alpha| \leq l_{0}}, \bar{f}^{\prime}(w)\right)((\chi \circ f)(z))^{i} \equiv 0 .
$$

From Lemma 4.4 and Lemma 4.5 (ii), we have the following formal identity

$$
\bar{\Phi}_{\theta^{\alpha}, \mu}^{\prime}\left(f(z), \bar{f}^{\prime}(w)\right)=\frac{V_{\alpha}^{\mu}\left(\left(\partial^{\beta} \bar{f}(w)\right)_{|\beta| \leq|\alpha|}, z, w\right)}{D^{2|\alpha|-1}(z, w)}, \text { in } \mathcal{M}
$$


for any $\alpha \in \mathbb{N}^{N}$, and $1 \leq \mu \leq c$. Thus, plugging this in (4.16), we obtain, for $(z, w) \in \mathcal{M}$,

$$
\sum_{i=0}^{k_{0}} s_{i}\left(\left(\frac{V_{\alpha}^{\mu}\left(\left(\partial^{\beta} \bar{f}(w)\right)_{|\beta| \leq|\alpha|}, z, w\right)}{D^{2|\alpha|-1}(z, w)}\right)_{1 \leq \mu \leq c,|\alpha| \leq l_{0}}, \bar{f}^{\prime}(w)\right)((\chi \circ f)(z))^{i} \equiv 0 .
$$

We claim that for $(z, w) \in \mathcal{M}$

$$
s_{k_{0}}\left(\left(\frac{V_{\alpha}^{\mu}\left(\left(\partial^{\beta} \bar{f}(w)\right)_{|\beta| \leq|\alpha|}, z, w\right)}{D^{2|\alpha|-1}(z, w)}\right)_{1 \leq \mu \leq c,|\alpha| \leq l_{0}}, \bar{f}^{\prime}(w)\right) \not \equiv 0 .
$$

Indeed, we have first to notice that, by definition, the left hand side of (4.18) is equal to

$$
s_{k_{0}}\left(\left(\bar{\Phi}_{\theta^{\alpha}, \mu}^{\prime}\left(f(z), \bar{f}^{\prime}(w)\right)\right)_{1 \leq \mu \leq c,|\alpha| \leq l_{0}}, \bar{f}^{\prime}(w)\right) .
$$

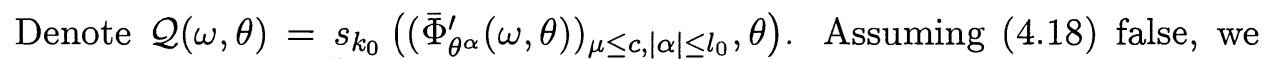
would get $\mathcal{Q}\left(f(z), \bar{f}^{\prime}(w)\right) \equiv 0$, for $(z, w) \in \mathcal{M}$. Since $f$ is nondegenerate, one can easily show that the rank of the formal holomorphic map $\mathcal{M} \ni(z, w) \mapsto$ $\left(f(z), \bar{f}^{\prime}(w)\right) \in \mathbb{C}^{2 n-c}$ is $2 n-c$. (By the rank of a formal holomorphic mapping $g(x)=\left(g_{1}(x), \ldots, g_{k}(x)\right)$, we mean its rank in the quotient field of $\mathbb{C}[[x]]$.) By standard arguments about formal power series, this implies that $\mathcal{Q}$ is identically zero as a formal power series, and hence, identically zero as a convergent one. This contradicts (4.15) and thus proves (4.18). To conclude the proof of Proposition 4.3, we observe the following. Since each $s_{i}, 0 \leq i \leq k_{0}$, is a polynomial, one sees that multiplying (4.17) by enough powers of $D(z, w)$, we have reached the desired conclusion in view of (i) and (ii) of Lemma 4.5. This finishes the proof of Proposition 4.3.

Remark 2. By Proposition 4.1, Proposition 4.3 also holds for any algebraic function $\chi$ belonging to the algebraic closure of the field generated by $\mathcal{C}$.

Remark 3. It is worth mentioning that, if, in Proposition $4.3, M^{\prime}$ is furthermore assumed to be holomorphically nondegenerate, then one can obtain a more precise statement. Indeed, when $M^{\prime}$ is holomorphically nondegenerate, by Theorem 4.2 , the algebraic closure of the field generated by the family $\mathcal{C}$ coincides with $\mathcal{F}_{n}$. Thus, in view of Remark 2, we can apply Proposition 4.3 to the algebraic functions $\chi(\omega)=\omega_{i}$, for $i=1, \ldots, n$, taken as coordinates. This gives the following proposition. (Recall also that by Remark 1, when $M^{\prime}$ is holomorphically nondegenerate, $l_{0}=l\left(M^{\prime}\right)$, the Levi-type of $M^{\prime}$.) 
Proposition 4.6. Let $M \subset \mathbb{C}^{n}$ be a real analytic generic submanifold through the origin and $M^{\prime} \subset \mathbb{C}^{n}$ be a real algebraic generic submanifold through the origin with the same $C R$ dimension. Let $f:(M, 0) \rightarrow\left(M^{\prime}, 0\right)$ be a formal nondegenerate $C R$ map and assume that $M^{\prime}$ is holomorphically nondegenerate. Then, for $j=1, \ldots, n$, there exists a positive integer $k_{j}$ (depending only on $M^{\prime}$ ) and a family of convergent power series $\delta_{i, j}=$ $\delta_{i, j}\left(\left(\Lambda_{\gamma}\right)_{|\gamma| \leq l\left(M^{\prime}\right)}, z, w\right) \in \mathbb{C}\left\{\left(\Lambda_{\gamma}-\partial^{\gamma} \bar{f}(0)\right)_{|\gamma| \leq l\left(M^{\prime}\right)}, z, w\right\}, i=0, \ldots, k_{j}$, such that the formal identity

$$
\sum_{i=0}^{k_{j}} \delta_{i, j}\left(\left(\partial^{\gamma} \bar{f}(w)\right)_{|\gamma| \leq l\left(M^{\prime}\right)}, z, w\right)\left(f_{j}(z)\right)^{i}=0
$$

holds for $(z, w) \in \mathcal{M}$, with $\delta_{k_{j}, j}\left(\left(\partial^{\gamma} \bar{f}(w)\right)_{|\gamma| \leq l\left(M^{\prime}\right)}, z, w\right) \not \equiv 0$ on $\mathcal{M}$.

Remark 4. In view of the works of Baouendi, Ebenfelt and Rothschild $[3,5,6]$, Proposition 4.3 can be viewed as a generalized reflection identity. We shall propose in the next section an algebraic interpretation of this identity, which can be compared to the work of Coupet, Pinchuk and Sukhov [10].

\section{A principle of analyticity for formal CR power series.}

Throughout this section, which is independent of $\S 4$, we shall consider one real analytic generic submanifold $M$, of CR dimension $N$ and of real codimension $c$ through the origin in $\mathbb{C}^{n}, n>1$. We shall also use the notations introduced for $M$ in $\S 3$. In particular, the complexification of $M$ is still denoted by $\mathcal{M}$. The purpose of $\S 5$ is to prove the following principle of convergence for formal CR functions. Recall that by a formal CR function, we mean a formal holomorphic power series.

Theorem 5.1. Let $M$ be a real analytic generic submanifold at $0 \in \mathbb{C}^{n}$. Let $h(z)$ be a holomorphic formal power series in $\mathbb{C}[[z]], z \in \mathbb{C}^{n}$. Assume that:

(i) $M$ is minimal at 0 ;

(ii) there exists a formal power series mapping $X(w)=\left(X_{1}(w), \ldots\right.$, $\left.X_{m}(w)\right) \in(\mathbb{C}[[w]])^{m}, w \in \mathbb{C}^{n}, X(0)=0$, and a family of convergent power series $\mathcal{U}_{j}(X, z, w) \in \mathbb{C}\{X, z, w\}, j=0, \ldots, l, l \in \mathbb{N}^{*}$, such that the relation

$$
\sum_{j=0}^{l} \mathcal{U}_{j}(X(w), z, w)(h(z))^{j}=0
$$


holds as a formal power series identity for $(z, w) \in \mathcal{M}$, and such that

$$
\mathcal{U}_{l}(X(w), z, w) \not \equiv 0, \text { for }(z, w) \in \mathcal{M} .
$$

Then $h(z)$ is convergent.

The proof of Theorem 5.1 will be divided in three distinct steps.

\subsection{Algebraic dependence of the jets.}

Proposition 5.2. Let $M$ be real analytic generic submanifold through the origin in $\mathbb{C}^{n}$ and $h(z)$ be a formal holomorphic power series in $z=$ $\left(z_{1}, \ldots, z_{n}\right)$. Assume that $h$ satisfies (ii) of Theorem 5.1. Then, for any multi-index $\mu \in \mathbb{N}^{n}$, there exists two positive integers $l(\mu), p(\mu)$, a family of convergent power series $\mathcal{U}_{i, \mu}\left(\left(\Lambda_{\gamma}\right)_{|\gamma| \leq|\mu|}, z, w\right) \in \mathbb{C}\left\{\left(\Lambda_{\gamma}-\right.\right.$ $\left.\left.\partial^{\gamma} X(0)\right)_{|\gamma| \leq|\mu|}, z, w\right\}, i=0, \ldots, l(\mu)$, such that the formal identity

$$
\sum_{i=0}^{l(\mu)} \mathcal{U}_{i, \mu}\left(\left(\partial^{\gamma} X(w)\right)_{|\gamma| \leq|\mu|}, z, w\right)\left(\partial^{\mu} h(z)\right)^{i}=0
$$

holds for $(z, w) \in \mathcal{M}$, and such that $\mathcal{U}_{l(\mu), \mu}\left(\left(\partial^{\gamma} X(w)\right)_{|\gamma| \leq|\mu|}, z, w\right) \not \equiv 0$, for $(z, w) \in \mathcal{M}$. Here, $X(w)$ is the formal power series mapping given by (ii) of Theorem 5.1.

Proof. Since $h(z)$ satisfies (ii) of Theorem 5.1, there exists a formal power series mapping $X(w)=\left(X_{1}(w), \ldots, X_{m}(w)\right) \in(\mathbb{C}[[w]])^{m}, w \in \mathbb{C}^{n}, X(0)=$ 0 , and a family of convergent power series $\mathcal{U}_{j}(X, z, w) \in \mathbb{C}\{X, z, w\}, j=$ $0, \ldots, l$, such that the formal identities (5.1) and (5.2) hold. For the proof of the proposition, we assume that the complexification of $M$ is given by $\mathcal{M}=\left\{(z, w) \in\left(\mathbb{C}^{2 n}, 0\right): w^{*}=\bar{\Phi}\left(z, w^{\prime}\right)\right\}$, with $\bar{\Phi}$ as defined in $\S 3.2$. (Recall that $w=\left(w^{\prime}, w^{*}\right) \in \mathbb{C}^{N} \times \mathbb{C}^{c}$.) It will be convenient to introduce for any integer $k$, a subring $\mathcal{A}_{k} \subset \mathbb{C}\left[\left[z, w^{\prime}\right]\right]$ which is defined as follows. Let

$$
\Pi_{X}^{k}: \mathbb{C}\left\{\left(\Lambda_{\gamma}-\partial^{\gamma} X(0)\right)_{|\gamma| \leq k}, z, w^{\prime}\right\} \rightarrow \mathbb{C}\left[\left[z, w^{\prime}\right]\right]
$$

be the substitution homomorphism defined by

$$
\left(\Lambda_{\gamma}\right)_{|\gamma| \leq k} \mapsto\left(\partial^{\gamma} X\left(w^{\prime}, \bar{\Phi}\left(z, w^{\prime}\right)\right)\right)_{|\gamma| \leq k}, z \mapsto z, w^{\prime} \mapsto w^{\prime} .
$$

$\mathcal{A}_{k}$ is, by definition, the ring image $\Pi_{X}^{k}\left(\mathbb{C}\left\{\left(\Lambda_{\gamma}-\partial^{\gamma} X(0)\right)_{|\gamma| \leq k}, z, w^{\prime}\right\}\right)$. Finally, we define $\mathcal{B}_{k}$ to be the quotient field of $\mathcal{A}_{k}$. The reader can now easily 
check that, to prove the proposition, it is equivalent to prove

$(*)_{\mu} \quad \partial^{\mu} h(z)$ is algebraic over the field $\mathcal{B}_{|\mu|}$,

for any $\mu \in \mathbb{N}^{n}$. We shall prove $(*)_{\mu}$ by induction on $|\mu|$. For $|\mu|=0,(*)_{0}$ follows from (5.1) and (5.2) and the definition of $\mathcal{B}_{0}$. Assume that $(*)_{\mu}$ holds for all $|\mu|=k$. This means precisely that, for any $\mu \in \mathbb{N}^{n}$ such that $|\mu|=k$, there exist two positive integers $l(\mu), p(\mu)$, a family of convergent power series $a_{i, \mu}\left(\left(\Lambda_{\gamma}\right)_{|\gamma| \leq|\mu|}, z, w^{\prime}\right) \in \mathbb{C}\left\{\left(\Lambda_{\gamma}-\partial^{\gamma} X(0)\right)_{|\gamma| \leq|\mu|}, z, w^{\prime}\right\}, i=0, \ldots, l(\mu)$, such that the formal identity

$$
\sum_{i=0}^{l(\mu)} a_{i, \mu}\left(\left(\partial^{\gamma} X\left(w^{\prime}, \bar{\Phi}\left(z, w^{\prime}\right)\right)\right)_{|\gamma| \leq|\mu|}, z, w^{\prime}\right)\left(\partial^{\mu} h(z)\right)^{i} \equiv 0
$$

holds in $\mathbb{C}\left[\left[z, w^{\prime}\right]\right]$, and such that

$$
a_{l(\mu), \mu}\left(\left(\partial^{\gamma} X\left(w^{\prime}, \bar{\Phi}\left(z, w^{\prime}\right)\right)\right)_{|\gamma| \leq|\mu|}, z, w^{\prime}\right) \not \equiv 0 .
$$

Moreover, we can choose $l(\mu)$ minimal satisfying a non-trivial relation such as (5.3). This implies that

$$
\sum_{j=1}^{l(\mu)} j a_{j, \mu}\left(\left(\partial^{\gamma} X\left(w^{\prime}, \bar{\Phi}\left(z, w^{\prime}\right)\right)\right)_{|\gamma| \leq|\mu|}, z, w^{\prime}\right)\left(\partial^{\mu} h(z)\right)^{j-1} \not \equiv 0,
$$

in $\mathbb{C}\left[\left[z, w^{\prime}\right]\right]$. In what follows, for $j=1, \ldots, n, 1_{j}$ is the multiindex of $\mathbb{N}^{n}$ with 1 at the $j$-th digit and 0 elsewhere. Applying $\partial_{z_{j}}$ for $j=1, \ldots, n$ to (5.3), we obtain

$$
\begin{array}{r}
\left(\partial^{\mu+1_{j}} h(z)\right) \sum_{j=1}^{l} j a_{j, \mu}\left(\left(\partial^{\gamma} X\left(w^{\prime}, \bar{\Phi}\left(z, w^{\prime}\right)\right)\right)_{|\gamma| \leq|\mu|}, z, w^{\prime}\right)\left(\partial^{\mu} h(z)\right)^{j-1} \\
\in \mathcal{A}_{k+1}\left[\partial^{\mu} h(z)\right]
\end{array}
$$

where $\mathcal{A}_{k+1}\left[\partial^{\mu} h(z)\right]$ is the subring of $\mathbb{C}\left[\left[z, w^{\prime}\right]\right]$ generated by $\mathcal{A}_{k+1}$ and $\partial^{\mu} h(z)$. By (5.5) and (5.6), we see that $\partial^{\mu+1_{j}} h(z)$ is algebraic over the field $\mathcal{B}_{k+1}\left(\partial^{\mu} h(z)\right)$, which is the subfield of Frac $\mathbb{C}\left[\left[z, w^{\prime}\right]\right]$ generated by $\mathcal{B}_{k+1}$ and $\partial^{\mu} h(z)$. Since $(*)_{\mu}$ holds, $\partial^{\mu} h(z)$ is algebraic over $\mathcal{B}_{k} \subset \mathcal{B}_{k+1}$, and thus, we see that $\partial^{\mu+1_{j}} h(z)$ is algebraic over $\mathcal{B}_{k+1}$ according to the transitivity of algebraicity over fields [28]. This shows that $(*)_{\nu}$ holds for all multiindeces $\nu \in \mathbb{N}^{n}$ such that $|\nu|=k+1$. This completes the proof of $(*)_{\mu}$ for all multiindeces $\mu \in \mathbb{N}^{n}$ and thus the proof of Proposition 5.2. 


\subsection{Non-trivial relations at the level of the Segre sets.}

In this section, we shall make use of the Segre sets mappings $v_{j}, j \in \mathbb{N}$, associated to $M$ as defined by (3.5) and (3.6). We shall also keep the notations introduced in $\S 3$ for $M$. Our main purpose here is to establish the following result.

Proposition 5.3. Under the assumptions and notations of Theorem 5.1, the following holds. For any multi-index $\mu \in \mathbb{N}^{n}$, and for any $d \in \mathbb{N}$, there exist two positive integers $\tau=\tau(\mu, d), p=p(\mu, d)$, and a family of convergent power series $g_{i \mu d}=g_{i \mu d}\left(\left(\Lambda_{\gamma}\right)_{|\gamma| \leq p}, z, w\right) \in \mathbb{C}\left\{\left(\Lambda_{\gamma}-\partial^{\gamma} X(0)\right)_{|\gamma| \leq p}, z, w\right\}$, $i=0, \ldots, \tau$, such that the formal identity

$$
\sum_{j=0}^{\tau} g_{j \mu d}\left(\left(\partial^{\gamma} X \circ \bar{v}_{d}\right)_{|\gamma| \leq p}, v_{d+1}, \bar{v}_{d}\right)\left(\partial^{\mu} h \circ v_{d+1}\right)^{j} \equiv 0
$$

holds in the ring of formal power series in $(d+1) N$ indeterminates, and such that $g_{\tau \mu d}\left(\left(\partial^{\gamma} X \circ \bar{v}_{d}\right)_{|\gamma| \leq p}, v_{d+1}, \bar{v}_{d}\right) \not \equiv 0$. Here, $X(w)$ is the formal power series mapping given by (ii) of Theorem 5.1 and $N$ is the $C R$ dimension of $M$.

Remark 5. If $M$ is a generic real analytic submanifold through the origin in $\mathbb{C}^{n}$, and $h(z)$ is a formal holomorphic power series in $z \in \mathbb{C}^{n}$ satisfying (ii) of Theorem 5.1, then, by applying Proposition 5.2, for any multi-index $\mu \in \mathbb{N}^{n}$, there exist two positive integers $l(\mu), p(\mu)$, a family of convergent power series $\mathcal{U}_{i, \mu}\left(\left(\Lambda_{\gamma}\right)_{|\gamma| \leq|\mu|}, z, w\right) \in \mathbb{C}\left\{\left(\Lambda_{\gamma}-\partial^{\gamma} X(0)\right)_{|\gamma| \leq|\mu|}, z, w\right\}, i=0, \ldots, l(\mu)$, such that the formal identity

$$
\sum_{i=0}^{l(\mu)} \mathcal{U}_{i, \mu}\left(\left(\partial^{\gamma} X(w)\right)_{|\gamma| \leq|\mu|}, z, w\right)\left(\partial^{\mu} h(z)\right)^{i}=0
$$

holds for $(z, w) \in \mathcal{M}$, and such that $\mathcal{U}_{l(\mu), \mu}\left(\left(\partial^{\gamma} X(w)\right)_{|\gamma| \leq|\mu|}, z, w\right) \not \equiv 0$ on $\mathcal{M}$. If, furthermore, $M$ is minimal at 0 , then for $d_{0}$ large enough, it follows from Theorem 3.1 and the definition of the Segre sets mappings given by (3.5) and (3.6) that, for $d \geq d_{0}$, the holomorphic map

$$
\left(\mathbb{C}^{d N}, 0\right) \ni\left(t_{1}, t_{2}, \ldots, t_{d}\right) \mapsto\left(v_{d+1}\left(t_{1}, t_{2}, \ldots\right), \bar{v}_{d}\left(t_{2}, \ldots\right)\right) \in \mathcal{M}
$$

is generically submersive. Thus, by elementary facts about formal power series, this implies that for $d \geq d_{0}$,

$$
\mathcal{U}_{l(\mu), \mu}\left(\left(\partial^{\gamma} X \circ \bar{v}_{d}\right)_{|\gamma| \leq|\mu|}, v_{d+1}, \bar{v}_{d}\right) \not \equiv 0 .
$$


This means that the algebraic relations

$$
\sum_{i=0}^{l(\mu)} \mathcal{U}_{i, \mu}\left(\left(\partial^{\gamma} X \circ \bar{v}_{d}\right)_{|\gamma| \leq|\mu|}, v_{d+1}, \bar{v}_{d}\right)\left(\partial^{\mu} h \circ v_{d}\right)^{i}=0
$$

will still be non-trivial for $d \geq d_{0}$. This proves Proposition 5.3 for $d \geq d_{0}$. However, in general, plugging $z=v_{d+1}$ and $w=\bar{v}_{d}$ in (5.7) for $d<d_{0}$, could lead to trivial relations. Thus, one has to work a little bit more to prove Proposition 5.3 for $d<d_{0}$.

For the proof of Proposition 5.3, we need to introduce the following definition, in which only the generic submanifold $M$ is involved.

Definition 5.1. Let $M$ be a generic real analytic submanifold through the origin, of $\mathrm{CR}$ dimension $N$, and $v_{k}, k \in \mathbb{N}$, the associated Segre sets mappings as defined by (3.5) and (3.6). Let $Y(w)=\left(Y_{1}(w), \ldots, Y_{r}(w)\right) \in$ $(\mathbb{C}[[w]])^{r}$ be a formal power series mapping in $w=\left(w_{1}, \ldots, w_{n}\right)$. Given $d \in \mathbb{N}$ and a formal power series $q(z) \in \mathbb{C}\left[\left[z_{1}, \ldots, z_{n}\right]\right]$, we say that $q$ satisfies property $\mathcal{P}(M, Y, d)$ if there exists a family of convergent power series $A_{j}\left(\Lambda_{0}, z, w\right) \in \mathbb{C}\left\{\left(\Lambda_{0}-Y(0), z, w\right\}, j=0, \ldots, p, p \in \mathbb{N}^{*}\right.$, such that the identity

$$
\sum_{j=0}^{p} A_{j}\left(Y \circ \bar{v}_{d}, v_{d+1}, \bar{v}_{d}\right)\left(q \circ v_{d+1}\right)^{j} \equiv 0
$$

holds in the ring of formal power series in $(d+1) N$ indeterminates and such that $A_{p}\left(Y \circ \bar{v}_{d}, v_{d+1}, \bar{v}_{d}\right) \not \equiv 0$.

We will need the following lemma to derive Proposition 5.3.

Lemma 5.4. Let $M$ be a real analytic generic submanifold through the origin in $\mathbb{C}^{n}$. Let $Y(w)=\left(Y_{1}(w), \ldots, Y_{r}(w)\right) \in(\mathbb{C}[[w]])^{r}$ be a formal power series mapping in $w=\left(w_{1}, \ldots, w_{n}\right)$. Let $d \in \mathbb{N}$ and $q(z) \in \mathbb{C}[[z]]$, $z=\left(z_{1}, \ldots, z_{n}\right)$. Then, if $q(z)$ satisfies property $\mathcal{P}(M, Y, d+2)$, there exists an integer $n_{0}$ (depending on $Y, q$ and $d$ ) such that $q(z)$ satisfies property $\mathcal{P}\left(M,\left(\partial^{\beta} Y\right)_{|\beta| \leq n_{0}}, d\right)$.

Proof of Lemma 5.4. Let $Y, q(z)$ and $d$ be as in the Lemma. We assume that $q(z)$ satisfies property $\mathcal{P}(M, Y, d+2)$. By definition, there exists a family of convergent power series $A_{j}\left(\Lambda_{0}, z, w\right) \in \mathbb{C}\left\{\left(\Lambda_{0}-Y(0), z, w\right\}, j=0, \ldots, p\right.$, 
$p \in \mathbb{N}^{*}$, such that the formal identity

$$
\sum_{j=0}^{p} A_{j}\left(Y \circ \bar{v}_{d+2}, v_{d+3}, \bar{v}_{d+2}\right)\left(q \circ v_{d+3}\right)^{j} \equiv 0
$$

holds and such that $A_{p}\left(Y \circ \bar{v}_{d+2}, v_{d+3}, \bar{v}_{d+2}\right) \not \equiv 0$. Here,

$$
\begin{gathered}
v_{d+3}=v_{d+3}\left(t_{1}, t_{2}, \ldots, t_{d+3}\right)=v_{d+3}\left(t_{1}, t^{\prime}\right)=v_{d+3}(t), \\
\bar{v}_{d+2}=\bar{v}_{d+2}\left(t_{2}, \ldots, t_{d+3}\right)=\bar{v}_{d+2}\left(t^{\prime}\right) .
\end{gathered}
$$

Thus, (5.8) holds in the ring $\mathbb{C}\left[\left[t_{1}, \ldots, t_{d+3}\right]\right]$. For simplicity of notations, we put, for $j=0, \ldots, p$,

$$
\Theta_{j}(t)=A_{j}\left(\left(\left(Y \circ \bar{v}_{d+2}\right)\left(t^{\prime}\right), v_{d+3}(t), \bar{v}_{d+2}\left(t^{\prime}\right)\right) .\right.
$$

Thus, (5.8) can be rewritten as

$$
\sum_{j=0}^{p} \Theta_{j}(t)\left(\left(q \circ v_{d+3}\right)(t)\right)^{j} \equiv 0, \text { with }
$$

$$
\Theta_{p}(t) \not \equiv 0, \text { in } \mathbb{C}[[t]] .
$$

Consider the set $\mathcal{E}$ defined by

$$
\left\{\alpha \in \mathbb{N}^{N}: \exists j \in\{1, \ldots, p\} \text {, such that }\left[\frac{\partial^{|\alpha|} \Theta_{j}}{\partial t_{1}^{\alpha}}(t)\right]_{t_{1}=t_{3}} \not \equiv 0, \text { in } \mathbb{C}\left[\left[t^{\prime}\right]\right]\right\} \text {. }
$$

Observe that by (5.11), there exists a multiindex $\alpha \in \mathbb{N}^{N}$ such that

$$
\left[\frac{\partial^{|\alpha|} \Theta_{p}}{\partial t_{1}^{\alpha}}(t)\right]_{t_{1}=t_{3}} \not \equiv 0
$$

in $\mathbb{C}\left[\left[t^{\prime}\right]\right]$. This implies that $\mathcal{E}$ is not empty. Let $\alpha^{0} \in \mathbb{N}^{N}$ such that $\left|\alpha^{0}\right|=$ $\min \{|\beta|: \beta \in \mathcal{E}\}$. Then, if we apply $\frac{\partial^{\left|\alpha^{0}\right|}}{\partial t_{1}^{\alpha^{0}}}$ to $(5.10)$, it follows from Leibniz's formula that

$$
\frac{\partial^{\left|\alpha^{0}\right|} \Theta_{0}}{\partial t_{1}^{\alpha^{0}}}(t)+\sum_{j=1}^{p} \frac{\partial^{\left|\alpha^{0}\right|} \Theta_{j}}{\partial t_{1}^{\alpha^{0}}}(t)\left(\left(q \circ v_{d+3}\right)(t)\right)^{j}=\sum_{\substack{\beta \in \mathbb{N}^{N},|\beta|<\left|\alpha^{0}\right| \\ 1 \leq j \leq p}} \frac{\partial^{\beta} \Theta_{j}}{\partial t_{1}^{\beta}}(t) \vartheta_{\beta, j}(t),
$$


where, for any $\beta, j, \vartheta_{\beta, j}(t) \in \mathbb{C}[[t]]$. By the choice of $\alpha^{0}$, we have, for $|\beta|<\left|\alpha^{0}\right|$

$$
\left[\frac{\partial^{\beta} \Theta_{j}}{\partial t_{1}^{\beta}}\left(t_{1}, t^{\prime}\right)\right]_{t_{1}=t_{3}} \equiv 0, \text { in } \mathbb{C}\left[\left[t^{\prime}\right]\right], j=1, \ldots, p .
$$

Thus, if we restrict equation (5.12) to $t_{1}=t_{3}$, we get by (5.13) the following identity in the ring $\mathbb{C}\left[\left[t^{\prime}\right]\right]$

$$
\sum_{j=0}^{p}\left[\frac{\partial^{\left|\alpha^{0}\right|} \Theta_{j}}{\partial t_{1}^{\alpha^{0}}}\left(t_{1}, t^{\prime}\right)\right]_{t_{1}=t_{3}}\left(\left(q \circ v_{d+3}\right)\left(t_{3}, t_{2}, t_{3}, \ldots, t_{d+3}\right)\right)^{j} \equiv 0 .
$$

Here again, for simplicity of notations, we put

$$
\Theta_{j}^{\prime}\left(t^{\prime}\right)=\left[\frac{\partial^{\left|\alpha^{0}\right|} \Theta_{j}}{\partial t_{1}^{\alpha^{0}}}\left(t_{1}, t^{\prime}\right)\right]_{t_{1}=t_{3}}
$$

We observe that, by the choice of $\alpha^{0}$, there exists $j \in\{1, \ldots, p\}$ such that $\Theta_{j}^{\prime}\left(t^{\prime}\right) \not \equiv 0$. Denote $m_{1}=\operatorname{Sup}\left\{j \in\{1, \ldots, p\}: \Theta_{j}^{\prime}\left(t^{\prime}\right) \not \equiv 0\right\}$. It follows from the reality condition (3.4) and the definition of the Segre sets mappings given by (3.5) and (3.6) that

$$
v_{d+3}\left(t_{3}, t_{2}, t_{3}, \ldots, t_{d+1}\right)=v_{d+1}\left(t_{3}, \ldots, t_{d+3}\right) .
$$

Thus, (5.14) reads as

$$
\sum_{j=0}^{m_{1}} \Theta_{j}^{\prime}\left(t^{\prime}\right)\left(\left(q \circ v_{d+1}\right)\left(t_{3}, \ldots, t_{d+3}\right)\right)^{j} \equiv 0
$$

with, moreover,

$$
\Theta_{m_{1}}^{\prime}\left(t^{\prime}\right) \not \equiv 0
$$

First case. $d \geq 1$. (5.17) implies that there exists $\beta^{0} \in \mathbb{N}^{N}$ such that

$$
\left[\frac{\partial^{\left|\beta^{0}\right|} \Theta_{m_{1}}^{\prime}}{\partial t_{2}^{\beta^{0}}}\left(t_{2}, t_{3}, \ldots, t_{d+3}\right)\right]_{t_{2}=t_{4}} \not \equiv 0 .
$$

Thus, applying $\frac{\partial^{\left|\beta^{0}\right|}}{\partial t_{2}^{\beta^{0}}}$ to (5.16) and after evaluation at $t_{2}=t_{4}$, we obtain in the ring $\mathbb{C}\left[\left[t_{3}, t_{4}, \ldots, t_{d+3}\right]\right]$

$$
\sum_{j=0}^{m_{1}}\left[\frac{\partial^{\beta^{0} \mid} \Theta_{j}^{\prime}}{\partial t_{2}^{\beta^{0}}}\left(t^{\prime}\right)\right]_{t_{2}=t_{4}}\left(\left(q \circ v_{d+1}\right)\left(t_{3}, \ldots, t_{d+3}\right)\right)^{j} \equiv 0
$$


We shall now see that (5.19) gives the statement of the Lemma. By definition of the Segre sets mappings given by (3.5) and (3.6) and by the definition of the $\Theta_{j}$ given by (5.9), we have, for $0 \leq j \leq m_{1}$,

$$
\begin{aligned}
\Theta_{j}\left(t_{1}, t^{\prime}\right) & =A_{j}\left(Y \circ \bar{v}_{d+2}\left(t^{\prime}\right), t_{1}, \Phi\left(\bar{v}_{d+2}\left(t^{\prime}\right), t_{1}\right), \bar{v}_{d+2}\left(t^{\prime}\right)\right) \\
& =G_{j}^{1}\left(\left(Y \circ \bar{v}_{d+2}\right)\left(t^{\prime}\right), \bar{v}_{d+2}\left(t^{\prime}\right), t_{1}\right),
\end{aligned}
$$

where $G_{j}^{1}\left(\Lambda_{0}, w, t_{1}\right) \in \mathbb{C}\left\{\Lambda_{0}-Y(0), w, t_{1}\right\}$. Using (5.15), we obtain

$$
\begin{aligned}
\Theta_{j}^{\prime}\left(t^{\prime}\right) & =\left[\frac{\partial^{\left|\alpha^{0}\right|} G_{j}^{1}}{\partial t_{1}^{\alpha^{0}}}\right]\left(\left(Y \circ \bar{v}_{d+2}\right)\left(t^{\prime}\right), \bar{v}_{d+2}\left(t^{\prime}\right), t_{3}\right) \\
& =\left[\frac{\partial^{\left|\alpha^{0}\right|} G_{j}^{1}}{\partial t_{1}^{\alpha^{0}}}\right]\left(\left(Y\left(t_{2}, \bar{\Phi}\left(v_{d+1}, t_{2}\right)\right), t_{2}, \bar{\Phi}\left(v_{d+1}, t_{2}\right), t_{3}\right) .\right.
\end{aligned}
$$

Here, $v_{d+1}=v_{d+1}\left(t_{3}, t_{4}, \ldots, t_{d+3}\right)$. As a consequence, we have

$$
\frac{\partial^{\left|\beta^{0}\right|} \Theta_{j}^{\prime}}{\partial t_{2}^{\beta^{0}}}\left(t^{\prime}\right)=G_{j}^{2}\left(\left(\partial^{\gamma} Y\left(t_{2}, \bar{\Phi}\left(v_{d+1}, t_{2}\right)\right)_{|\gamma| \leq\left|\beta^{0}\right|}, v_{d+1}, t_{2}, t_{3}\right),\right.
$$

where $G_{j}^{2}=G_{j}^{2}\left(\left(\Lambda_{\gamma}\right)_{|\gamma| \leq\left|\beta^{0}\right|}, w, t_{2}, t_{3}\right) \in \mathbb{C}\left\{\left(\Lambda_{\gamma}-\partial^{\gamma} Y(0)\right)_{|\gamma| \leq\left|\beta^{0}\right|}, w, t_{2}, t_{3}\right\}$. Here again, by $(3.4),(3.5)$ and $(3.6)$, we have $\bar{v}_{d+2}\left(t_{4}, t_{3}, t_{4}, t_{5}, \ldots\right)=$ $\bar{v}_{d}\left(t_{4}, t_{5}, \ldots\right)$, and thus

$$
\begin{aligned}
& {\left[\frac{\partial^{\mid \beta^{0}} \Theta_{j}^{\prime}}{\partial t_{2}^{\beta^{0}}}\left(t^{\prime}\right)\right]_{t_{2}=t_{4}}} \\
& =G_{j}^{2}\left(\left(\left(\partial^{\gamma} Y \circ \bar{v}_{d+2}\right)\left(t_{4}, t_{3}, t_{4}, \ldots\right)\right)_{|\gamma| \leq\left|\beta^{0}\right|}, v_{d+1}\left(t_{3}, t_{4}, \ldots\right), t_{4}, t_{3}\right) \\
& =G_{j}^{2}\left(\left(\left(\partial^{\gamma} Y \circ \bar{v}_{d}\right)\left(t_{4}, \ldots, t_{d+3}\right)\right)_{|\gamma| \leq\left|\beta^{0}\right|}, v_{d+1}\left(t_{3}, t_{4}, \ldots\right), t_{4}, t_{3}\right) \\
& =B_{j}\left(\left(\partial^{\gamma} Y \circ \bar{v}_{d}\right)_{|\gamma| \leq\left|\beta^{0}\right|}, v_{d+1}, \bar{v}_{d}\right),
\end{aligned}
$$

where $B_{j}$ for $j=0, \ldots, m_{1}$, is a convergent power series in its arguments. Consequently, from (5.19) and (5.20) we have the relation

$$
\sum_{j=0}^{m_{1}} B_{j}\left(\left(\partial^{\gamma} Y \circ \bar{v}_{d}\right)_{|\gamma| \leq\left|\beta^{0}\right|}, v_{d+1}, \bar{v}_{d}\right)\left(q \circ v_{d+1}\right)^{j} \equiv 0 \text {, in } \mathbb{C}\left[\left[t_{3}, \ldots, t_{d+3}\right]\right],
$$

which is non-trivial according to (5.18) and (5.20). In conclusion, $q(z)$ satisfies property $\mathcal{P}\left(M,\left(\partial^{\gamma} Y\right)_{|\gamma| \leq\left|\beta^{0}\right|}, d\right)$. 
Second case. $d=0$. In this case, almost the same procedure used in the case $d \geq 1$ can be applied. Indeed, by (5.17), we have $\Theta_{m_{1}}^{\prime}\left(t_{2}, t_{3}\right) \not \equiv 0$ and therefore, there exists a multi-index $\varrho^{0} \in \mathbb{N}^{N}$ such that

$$
\left[\frac{\partial^{\left|\varrho^{0}\right|} \Theta_{m_{1}}^{\prime}}{\partial t_{2}^{\varrho^{0}}}\left(t_{2}, t_{3}\right)\right]_{t_{2}=0} \not \equiv 0
$$

Thus, applying $\frac{\partial^{\left|\varrho^{0}\right|}}{\partial t_{2}^{\varrho^{0}}}$ to $(5.16)$ and after evaluation at $t_{2}=0$, we obtain in the ring $\mathbb{C}\left[\left[t_{3}\right]\right]$

$$
\sum_{j=0}^{m_{1}}\left[\frac{\partial^{\left|\varrho^{0}\right|} \Theta_{j}^{\prime}}{\partial t_{2}^{\varrho^{0}}}\left(t_{2}, t_{3}\right)\right]_{t_{2}=0}\left(\left(q \circ v_{1}\right)\left(t_{3}\right)\right)^{j} \equiv 0 .
$$

As in the case $d \geq 1$, we have for $j=1, \ldots, m_{1}$,

$$
\frac{\partial^{\left|\varrho^{0}\right|} \Theta_{j}^{\prime}}{\partial t_{2}^{\varrho^{0}}}\left(t_{2}, t_{3}\right)=G_{j}^{3}\left(\left(\partial^{\gamma} Y\left(t_{2}, \bar{\Phi}\left(v_{1}\left(t_{3}\right), t_{2}\right)\right)_{|\gamma| \leq\left|\varrho^{0}\right|}, v_{1}\left(t_{3}\right), t_{2}, t_{3}\right),\right.
$$

where $G_{j}^{3}=G_{j}^{3}\left(\left(\Lambda_{\gamma}\right)_{|\gamma| \leq\left|\varrho^{0}\right|}, w, t_{2}, t_{3}\right) \in \mathbb{C}\left\{\left(\Lambda_{\gamma}-\partial^{\gamma} Y(0)\right)_{|\gamma| \leq\left|\varrho^{0}\right|}, w, t_{2}, t_{3}\right\}$. By the normality of the coordinates for $M$, we have for $j=0, \ldots, m_{1}$,

$$
\left[\frac{\partial^{\left|\varrho^{0}\right|} \Theta_{j}^{\prime}}{\partial t_{2}^{\varrho^{0}}}\left(t_{2}, t_{3}\right)\right]_{t_{2}=0}=G_{j}^{3}\left(\left(\partial^{\gamma} Y(0)\right)_{|\gamma| \leq\left|\varrho^{0}\right|}, v_{1}\left(t_{3}\right), 0, t_{3}\right) .
$$

We leave it to the reader to check that, similarly to the case $d \geq 1,(5.23)$, (5.22) and (5.21) give the desired statement of the lemma for $d=0$, i.e., that $q(z)$ satisfies property $\mathcal{P}\left(M,\left(\partial^{\gamma} Y\right)_{|\gamma| \leq\left|\beta^{0}\right|}, 0\right)$. This completes the proof of Lemma 5.4.

Proof of Proposition 5.3. Let $\mu \in \mathbb{N}^{n}$. Since $h(z)$ satisfies (ii) of Theorem 5.1, by Proposition 5.2, there exist two positive integers $l(\mu), p(\mu)$ and a family of convergent power series $\mathcal{U}_{i, \mu}\left(\left(\Lambda_{\gamma}\right)_{|\gamma| \leq|\mu|}, z, w\right) \in \mathbb{C}\left\{\left(\Lambda_{\gamma}-\right.\right.$ $\left.\left.\partial^{\gamma} X(0)\right)_{|\gamma| \leq|\mu|}, z, w\right\}, i=0, \ldots, l(\mu)$, such that the formal identity

$$
\sum_{i=0}^{l(\mu)} \mathcal{U}_{i, \mu}\left(\left(\partial^{\gamma} X(w)\right)_{|\gamma| \leq|\mu|}, z, w\right)\left(\partial^{\mu} h(z)\right)^{i}=0
$$


holds on $\mathcal{M}$ and such that

$$
\mathcal{U}_{l(\mu), \mu}\left(\left(\partial^{\gamma} X(w)\right)_{|\gamma| \leq|\mu|}, z, w\right) \neq 0
$$

for $(z, w) \in \mathcal{M}$. Notice that to prove the proposition we have to show that for any $d \in \mathbb{N}$, there exists $p=p(\mu, d)$ such that $\partial^{\mu} h(z)$ satisfies property $\mathcal{P}\left(M,\left(\partial^{\beta} X\right)_{|\beta| \leq p}, d\right)$. Since $M$ is minimal at $0 \in M$, it follows from Theorem 3.1 and the definition of the Segre sets mappings given by (3.5) and (3.6) that there exists $d_{0} \in \mathbb{N}$ (which can be assumed to be even) such that the holomorphic map $\mathbb{C}^{\left(d_{0}+1\right) N} \ni\left(t_{1}, \ldots, t_{d_{0}+1}\right) \mapsto$ $\left(v_{d_{0}+1}\left(t_{1}, t_{2}, \ldots, t_{d_{0}+1}\right), \bar{v}_{d_{0}}\left(t_{2}, \ldots, t_{d_{0}+1}\right)\right) \in \mathcal{M}$ is generically submersive. By elementary facts about formal power series and by (5.25), this implies that

$$
\mathcal{U}_{l(\mu), \mu}\left(\left(\partial^{\gamma} X \circ \bar{v}_{d_{0}}\right)_{|\gamma| \leq|\mu|}, v_{d_{0}+1}, \bar{v}_{d_{0}}\right) \not \equiv 0 .
$$

This means that the following algebraic relation obtained from (5.24),

$$
\sum_{i=0}^{l(\mu)} \mathcal{U}_{i, \mu}\left(\left(\partial^{\gamma} X \circ \bar{v}_{d_{0}}\right)_{|\gamma| \leq|\mu|}, v_{d_{0}+1}, \bar{v}_{d_{0}}\right)\left(\left(\partial^{\mu} h\right) \circ v_{d_{0}+1}\right)^{i} \equiv 0,
$$

is still non-trivial, i.e., that $\partial^{\mu} h(z)$ satisfies property $\mathcal{P}\left(M,\left(\partial^{\beta} X\right)_{|\beta| \leq|\mu|}, d_{0}\right)$. (Observe that in Remark 5, we have shown that $\partial^{\mu} h(z)$ satisfies property $\mathcal{P}\left(M,\left(\partial^{\beta} X\right)_{|\beta| \leq|\mu|}, d\right)$ for any $d \geq d_{0}$.) Applying Lemma 5.4 to $q(z)=$ $\partial^{\mu} h(z)$ and $Y=\left(\partial^{\beta} X\right)_{|\beta| \leq|\mu|}$, we obtain that there exists $p\left(\mu, d_{0}-2\right) \in \mathbb{N}$ such that $\partial^{\mu} h(z)$ satisfies property $\mathcal{P}\left(M,\left(\partial^{\beta} X\right)_{|\beta| \leq p\left(\mu, d_{0}-2\right)}, d_{0}-2\right)$. Hence, using inductively Lemma 5.4 , we obtain that for any even number $0 \leq$ $d \leq d_{0}$, there exists $p(\mu, d) \in \mathbb{N}$ such that $\partial^{\mu} h(z)$ satisfies property $\mathcal{P}\left(M,\left(\partial^{\beta} X\right)_{|\beta| \leq p(\mu, d)}, d\right)$. Since by Remark $5, \partial^{\mu} h(z)$ satisfies also property $\mathcal{P}\left(M,\left(\partial^{\beta} X\right)_{|\beta| \leq|\mu|}, d_{0}+1\right)$, we can again, in the same way, use Lemma 5.4 to conclude that for any odd number $1 \leq d \leq d_{0}$, there exists $p(\mu, d) \in \mathbb{N}$ such that $\partial^{\mu} h(z)$ satisfies property $\mathcal{P}\left(M,\left(\partial^{\beta} X\right)_{|\beta| \leq p(\mu, d)}, d\right)$. This completes the proof of Proposition 5.3.

\subsection{Propagation procedure.}

We prove here the last proposition needed for the proof of Theorem 5.1.

Proposition 5.5. Let $M$ be a generic real analytic submanifold through the origin, and $v_{k}, k \in \mathbb{N}$, the associated Segre sets mappings as defined by (3.5) and (3.6). Let $h(z) \in \mathbb{C}\left[\left[z_{1}, \ldots, z_{n}\right]\right]$ and $d \in \mathbb{N}$. Let $\mu \in \mathbb{N}^{n}$ and assume that 
there exists $Y_{\mu d}(w)$, a formal power series mapping in $w=\left(w_{1}, \ldots, w_{n}\right)$, such that $\partial^{\mu} h(z)$ satisfies property $\mathcal{P}\left(M, Y_{\mu d}, d+2\right)$ as defined in Definition 5.1. Then the following holds. If for any multiindices $\nu \in \mathbb{N}^{n}, \partial^{\nu} h \circ v_{d+1}$ is convergent, then $\partial^{\mu} h \circ v_{d+3}$ is convergent.

For the proof of Proposition 5.5, we need the following two lemmas which are both consequences of the Artin approximation theorem [1]. We refer the reader to [21] for the proof of the first one and to $[6,21]$ for the proof of the second one.

Lemma 5.6. Let $\mathcal{T}(x, u)=\left(\mathcal{T}_{1}(x, u), \ldots, \mathcal{T}_{r}(x, u)\right) \in(\mathbb{C}[[x, u]])^{r}, x \in \mathbb{C}^{q}$, $u \in \mathbb{C}^{s}$, with $\mathcal{T}(0)=0$. Assume that $\mathcal{T}(x, u)$ satisfies an identity in the ring $\mathbb{C}[[x, u, y]], y \in \mathbb{C}^{q}$, of the form

$$
\varphi(\mathcal{T}(x, u) ; x, u, y)=0
$$

where $\varphi \in \mathbb{C}[[W, x, u, y]]$ with $W \in \mathbb{C}^{r}$. Assume, furthermore, that for any multi-index $\beta \in \mathbb{N}^{q}$, the formal power series $\left[\frac{\partial^{|\beta|} \varphi}{\partial y^{\beta}}(W ; x, u, y)\right]_{y=x} i s$ convergent, i.e., belongs to $\mathbb{C}\{W, x, u\}$. Then, for any given positive integer $e$, there exists an $r$-tuple of convergent power series $\mathcal{T}^{e}(x, u) \in(\mathbb{C}\{x, u\})^{r}$ such that $\varphi\left(\mathcal{T}^{e}(x, u) ; x, u, y\right)=0$ in $\mathbb{C}[[x, u, y]]$ and such that $\mathcal{T}^{e}(x, u)$ agrees up to order $e$ (at 0$)$ with $\mathcal{T}(x, u)$.

Lemma 5.7. Any formal power series in $r$ indeterminates, which is algebraic over the field of meromorphic functions (in $r$ variables), must be convergent.

Remark 6. We would like to mention that the use of the Artin approximation theorem is not a novelty in the study of many mapping problems ( $c f$. $[11,8,20,6,21]$ as well as many other articles).

Proof of Proposition 5.5. Let $\mu \in \mathbb{N}^{n}$ and $d \in \mathbb{N}$ be as in the statement of the proposition. We assume that for any multiindex $\nu \in \mathbb{N}^{n}, \partial^{\nu} h \circ v_{d+1}$ is convergent. By assumption, there exists $Y_{\mu d}(w) \in(\mathbb{C}[[w]])^{r}, r=r(\mu, d) \in$ $\mathbb{N}^{*}$, a formal power series mapping in $w=\left(w_{1}, \ldots, w_{n}\right)$ such that $\partial^{\mu} h(z)$ satisfies property $\mathcal{P}\left(M, Y_{\mu d}, d+2\right)$. By definition, this means that there exists a family of convergent power series $A_{j}=A_{j}\left(\Lambda_{0}, z, w\right) \in \mathbb{C}\left\{\Lambda_{0}-Y_{\mu d}(0), z, w\right\}$, 
$j=0, \ldots, k, k=k(\mu, d)$, such that the formal power series identity

$$
\sum_{j=0}^{k} A_{j}\left(\left(Y_{\mu d} \circ \bar{v}_{d+2}\right)\left(t^{\prime}\right), v_{d+3}(t), \bar{v}_{d+2}\left(t^{\prime}\right)\right)\left(\left(\left(\partial^{\mu} h\right) \circ v_{d+3}\right)(t)\right)^{j} \equiv 0
$$

holds in $\mathbb{C}[[t]]$, with $t=\left(t_{1}, t^{\prime}\right)=\left(t_{1}, t_{2}, \ldots, t_{d+3}\right)$ and such that

$$
A_{k}\left(\left(Y_{\mu d} \circ \bar{v}_{d+2}\right)\left(t^{\prime}\right), v_{d+3}(t), \bar{v}_{d+2}\left(t^{\prime}\right)\right) \not \equiv 0 .
$$

We would like to apply Lemma 5.6 with $y=t_{1}, x=t_{3}, u=$ $\left(t_{2}, t_{4}, t_{5}, \ldots, t_{d+3}\right), \mathcal{T}(x, u)=\left(Y_{\mu d} \circ \bar{v}_{d+2}\right)\left(t_{2}, \ldots, t_{d+3}\right)-Y_{\mu d}(0), W=\Lambda_{0}^{\prime}$ $\left(\Lambda_{0}^{\prime} \in \mathbb{C}^{r}\right)$ and

$$
\begin{aligned}
& \varphi\left(\Lambda_{0}^{\prime} ; t_{3},\left(t_{2}, t_{4}, t_{5}, \ldots, t_{d+3}\right), t_{1}\right) \\
& =\sum_{j=0}^{k} A_{j}\left(\Lambda_{0}^{\prime}+Y_{\mu d}(0), v_{d+3}(t), \bar{v}_{d+2}\left(t^{\prime}\right)\right)\left(\left(\left(\partial^{\mu} h\right) \circ v_{d+3}\right)(t)\right)^{j} .
\end{aligned}
$$

For this, one has to check that any derivative with respect to $t_{1}$ of $\varphi$ evaluated at $t_{1}=t_{3}$ is in fact convergent with respect to the variables $\Lambda_{0}^{\prime}$ and $t^{\prime}$. Because of the analyticity of the functions $A_{i}, i=0, \ldots, k$ (and of the Segre sets mappings), we see that we have only to consider the derivatives of $\left[\partial^{\mu} h \mathrm{o}\right.$ $\left.v_{d+3}(t)\right]^{j}$, for $j=0, \ldots, l$, evaluated at $t_{1}=t_{3}$. These derivatives involve analytic terms coming for the differentiation of $v_{d+3}$ (which are convergent) and products involving powers of derivatives of $h$ evaluated at $t_{1}=t_{3}$. Let $\left[\left(\partial^{\gamma} h\right) \circ v_{d+3}(t)\right]_{t_{1}=t_{3}}$ be such a derivative for some $\gamma \in \mathbb{N}^{n}$. By the reality condition (3.4) and by (3.5) and (3.6), we have

$$
v_{d+3}\left(t_{3}, t_{2}, t_{3}, t_{4}, \ldots, t_{d+3}\right)=v_{d+1}\left(t_{3}, t_{4}, \ldots, t_{d+3}\right) .
$$

Thus, $\left[\left(\partial^{\gamma} h\right) \circ v_{d+3}(t)\right]_{t_{1}=t_{3}}=\left(\left(\partial^{\gamma} h\right) \circ v_{d+1}\right)\left(t_{3}, \ldots, t_{d+3}\right)$ which is convergent by our hypothesis. As a consequence, $\varphi$ satifies the assumptions of Lemma 5.6. Thus, by applying that Lemma, one obtains for any positive integer $e$, a convergent power series mapping $\mathcal{T}^{e}\left(t^{\prime}\right)$, which agrees up to order $e$ with $\left(Y_{\mu d} \circ \bar{v}_{d+2}\right)\left(t^{\prime}\right)$ and such that

$$
\sum_{j=0}^{k} A_{j}\left(\mathcal{T}^{e}\left(t^{\prime}\right), v_{d+3}(t), \bar{v}_{d+2}\left(t^{\prime}\right)\right)\left(\left(\left(\partial^{\mu} h\right) \circ v_{d+3}\right)(t)\right)^{j} \equiv 0 .
$$

Observe that (5.28) implies that, for $e$ large enough, say $e=e_{0}$, the following condition will be satisfied

$$
A_{k}\left(\mathcal{T}^{e_{0}}\left(t^{\prime}\right), v_{d+3}(t), \bar{v}_{d+2}\left(t^{\prime}\right)\right) \not \equiv 0
$$

in $\mathbb{C}[[t]]$. This allows one to apply Lemma 5.7 to conclude that $\partial^{\mu} h \circ v_{d+3}$ is convergent. 


\subsection{Completion of the proof of Theorem 5.1.}

Let $h(z)$ be the formal power series of the Theorem and $X(w)$ the associated formal power series mapping given by (ii). By Proposition 5.3, for any multi-index $\mu \in \mathbb{N}^{n}$, and for any $d \in \mathbb{N}$, there exists two positive integers $\tau=\tau(\mu, d), p=p(\mu, d)$, and a family of convergent power series $g_{i \mu d}=$ $g_{i \mu d}\left(\left(\Lambda_{\gamma}\right)_{|\gamma| \leq p}, z, w\right) \in \mathbb{C}\left\{\left(\Lambda_{\gamma}-\partial^{\gamma} X(0)\right)_{|\gamma| \leq p}, z, w\right\}, i=0, \ldots, \tau$, such that the identity

$$
\sum_{j=0}^{\tau} g_{j \mu d}\left(\left(\partial^{\gamma} X \circ \bar{v}_{d}\right)_{|\gamma| \leq p}, v_{d+1}, \bar{v}_{d}\right)\left(\left(\partial^{\mu} h\right) \circ v_{d+1}\right)^{j} \equiv 0
$$

holds in $\mathbb{C}[[t]]$ where $t=\left(t_{1}, \ldots, t_{d+1}\right) \in \mathbb{C}^{(d+1) N}$, and with the additional nondegeneracy condition $g_{\tau \mu d}\left(\left(\partial^{\gamma} X \circ \bar{v}_{d}\right)_{|\gamma| \leq p}, v_{d+1}, \bar{v}_{d}\right) \not \equiv 0$. In view of Definition 5.1, this means that, for any multiindex $\mu \in \mathbb{N}^{n}$ and for any $d \in \mathbb{N}$, $\partial^{\mu} h(z)$ satisfies property $\mathcal{P}\left(M,\left(\partial^{\gamma} X\right)_{|\gamma| \leq p(\mu, d)}, d\right)$. Observe first that since for any $\nu \in \mathbb{N}^{n}, \partial^{\nu} h(z)$ satisfie property $\mathcal{P}\left(M,\left(\partial^{\gamma} X\right)_{|\gamma| \leq p(\nu, 0)}, 0\right)$, it follows from Lemma 5.7 (and from Definition 5.1) that $\partial^{\nu} h \circ v_{1}$ is convergent for any multiindex $\nu \in \mathbb{N}^{n}$. From this and the fact that $\partial^{\mu} h(z)$ satisfies property $\mathcal{P}\left(M,\left(\partial^{\gamma} X\right)_{|\gamma| \leq p(\mu, 2)}, 2\right)$, it follows from Proposition 5.5 that $\partial^{\mu} h \circ v_{3}$ is convergent, for all multiindices $\mu \in \mathbb{N}^{n}$. Thus, by induction, we see that Proposition 5.5 gives that for any odd number $d$, and for any multiindex $\mu \in \mathbb{N}^{n}$, $\partial^{\mu} h \circ v_{d}$ is convergent. Choose $d_{0} \in \mathbb{N}$ satisfying the statement of Theorem 3.1. Without loss of generality, $d_{0}$ can be assumed to be odd. By the previous considerations, we know that $h \circ v_{d_{0}}$ is convergent in some neighborhood $U$ of $0 \in \mathbb{C}^{d_{0} N}$. By Theorem 3.1, there exists $\left(t_{1}^{0}, \ldots, t_{d_{0}}^{0}\right) \in U$ such that $v_{d_{0}}\left(t_{1}^{0}, \ldots, t_{d_{0}}^{0}\right)=0$ and such that $v_{d_{0}}$ is submersive at $\left(t_{1}^{0}, \ldots, t_{d_{0}}^{0}\right)$. Thus, we may apply the rank theorem to conclude that $v_{d_{0}}$ has a right convergent inverse $\Theta(z) \in(\mathbb{C}\{z\})^{d_{0} N}$ defined near $0 \in \mathbb{C}^{n}$ such that $\Theta(0)=\left(t_{1}^{0}, \ldots, t_{d_{0}}^{0}\right)$ and such that $v_{d_{0}} \circ \Theta(z)=z$. This implies that $h(z)$ is convergent. The proof of Theorem 5.1 is complete.

\section{Proofs of Theorem 2.1 and Theorem 2.2 .}

Proof of Theorem 2.1. Recall that $M^{\prime}$ is given near 0 by (4.2) and that $\mathcal{C}$ is the family of algebraic holomorphic functions (in $n$ variables) defined by (4.5) and constructed from $M^{\prime}$. By Proposition 4.3, for any $\chi \in \mathcal{C}$, there exists $l_{0} \in \mathbb{N}^{*}$, a positive integer $k_{0}$ and a family of convergent power series $\delta_{i}=\delta_{i}\left(\left(\Lambda_{\gamma}\right)_{|\gamma| \leq l_{0}}, z, w\right) \in \mathbb{C}\left\{\left(\Lambda_{\gamma}-\partial^{\gamma} \bar{f}(0)\right)_{|\gamma| \leq l_{0}}, z, w\right\}, i=0, \ldots, k_{0}$, such 
that the formal power series identity

$$
\sum_{i=0}^{k_{0}} \delta_{i}\left(\left(\partial^{\gamma} \bar{f}(w)\right)_{|\gamma| \leq l_{0}}, z, w\right)((\chi \circ f)(z))^{i}=0
$$

holds for $(z, w) \in \mathcal{M}$ such that $\delta_{k_{0}}\left(\left(\partial^{\gamma} \bar{f}(w)\right)_{|\gamma| \leq l_{0}}, z, w\right) \not \equiv 0$ in $\mathcal{M}$. Since $M$ is minimal at 0 and $\chi \circ f$ satisfies (ii) of Theorem 5.1 by (6.1), we may apply that theorem to conclude that $\chi \circ f$ is convergent. In other words, for any $\alpha \in \mathbb{N}^{N}$ and for any $1 \leq \nu \leq c, q_{\alpha, \nu} \circ f$ is convergent. To conclude the proof of Theorem 2.1, we have to show that this implies that the reflection mapping

$$
\mathbb{C}^{n} \times \mathbb{C}^{N} \ni(z, \theta) \mapsto \bar{\Phi}^{\prime}(f(z), \theta) \in \mathbb{C}^{c}
$$

is convergent. To see this, it suffices to observe that since $M^{\prime}$ is real algebraic, the map $\mathbb{C}^{n} \times \mathbb{C}^{N} \ni(\zeta, \theta) \mapsto \bar{\Phi}_{\nu}^{\prime}(\omega, \theta) \in \mathbb{C}, 1 \leq \nu \leq c$, is algebraic, and thus, an approximation argument similar to the one used in the proof of Proposition 1 from [20] shows that for any $\nu \in\{1, \ldots, c\}, \bar{\Phi}_{\nu}^{\prime}(\omega, \theta)$ is algebraic over the field generated by $\mathbb{C}$ and the family of algebraic functions $\mathcal{C}$ and $\theta$. Since $f$ is nondegenerate, this implies that the formal power series $\mathbb{C}^{n} \times \mathbb{C}^{N} \ni(z, \theta) \mapsto \bar{\Phi}_{\nu}^{\prime}(f(z), \theta)$ is algebraic over the field generated by $\mathbb{C}$, the family of formal power series $\mathcal{C}_{f}=\left(\left(q_{\beta, \nu} \circ f\right)(z)\right)_{\beta \in \mathbb{N} N}$ and $\theta$. But since the family $\mathcal{C}_{f}$ is a family of convergent power series, Lemma 5.7 implies that the formal power series $\bar{\Phi}_{\nu}^{\prime}(f(z), \theta)$ is actually convergent for any $\nu \in\{1, \ldots, c\}$. This completes the proof of Theorem 2.1.

Remark 7. If, in Theorem 2.1, the target manifold is given in normal coordinates i.e., if $\bar{\Phi}^{\prime}(\omega, 0)=\omega^{*}$ where $\bar{\Phi}^{\prime}$ is given by $(4.2)$, then the following holds. The normal components $f^{*} \in \mathbb{C}^{c}$ (as in (4.7)) of a formal nondegenerate $\mathrm{CR}$ map $f$ from a real analytic generic submanifold into a real algebraic one are necessarily convergent provided that the source manifold is minimal. Indeed, this follows from Theorem 2.1 by taking $\theta=0$.

Proof of Theorem 2.2. By the Taylor expansion (4.3) and by Theorem 2.1, we know that for any $\beta \in \mathbb{N}^{N}$ and any $1 \leq \nu \leq c, q_{\beta, \nu} \circ f$ is convergent. Equivalently, we have the convergence of $\chi \circ f$ for any algebraic function $\chi \in \mathcal{C}$, where $\mathcal{C}$ is the family of algebraic functions defined by (4.5). Observe that since $f$ is nondegenerate, Lemma 5.7 implies that for any algebraic holomorphic function $q=q(\omega)$ in the algebraic closure of the field generated by the family $\mathcal{C}, q \circ f$ must also be convergent. To conclude that $f$ 
is convergent when $M^{\prime}$ is holomorphically nondegenerate, it suffices to apply Theorem 4.2 (iii) which states that this algebraic closure, in that case, coincides with all the field of algebraic functions $\mathcal{F}_{n}$.

\section{Remarks concerning Theorem 2.2.}

The purpose of this section is to show how the convergence result given by Theorem 2.2 can be derived from the arguments of $\S 5$ more simply than the arguments given in $\S 6$. Thus, let $f:(M, 0) \rightarrow\left(M^{\prime}, 0\right)$ be a formal nondegenerate CR map from a real analytic generic submanifold into a real algebraic one. We also assume that $M^{\prime}$ is holomorphically nondegenerate. Then, by Proposition 4.6, we know that for each component $f_{j}$ of $f, j=$ $1, \ldots, n$, there exists a positive integer $k_{j}$ and a family of convergent power series $\delta_{i, j}=\delta_{i, j}\left(\left(\Lambda_{\gamma}\right)_{|\gamma| \leq l\left(M^{\prime}\right)}, z, w\right) \in \mathbb{C}\left\{\left(\Lambda_{\gamma}-\partial^{\gamma} \bar{f}(0)\right)_{|\gamma| \leq l\left(M^{\prime}\right)}, z, w\right\}, i=$ $0, \ldots, k_{j}$, such that the formal identity

$$
\sum_{i=0}^{k_{j}} \delta_{i, j}\left(\left(\partial^{\gamma} \bar{f}(w)\right)_{|\gamma| \leq l\left(M^{\prime}\right)}, z, w\right)\left(f_{j}(z)\right)^{i}=0
$$

holds for $(z, w) \in \mathcal{M}$, with $\delta_{k_{j}, j}\left(\left(\partial^{\gamma} \bar{f}(w)\right)_{|\gamma| \leq l\left(M^{\prime}\right)}, z, w\right) \not \equiv 0$ on $\mathcal{M}$. Here, we recall that $l\left(M^{\prime}\right)$ is the Levi-type of $M^{\prime}$ as in Remark 1 and that $\mathcal{M}$ is the complexification of $M$ as defined in §3.1. Equation (7.1) means that for each $j=1, \ldots, n, f_{j}(z)$ satisfies the statement (ii) of Theorem 5.1, with associated formal power series mapping $X(w)=\left(\partial^{\gamma} \bar{f}(w)\right)_{|\gamma| \leq l\left(M^{\prime}\right)}$. Thus, if we apply Proposition 5.2 to $h(z)=f_{j}(z)\left(\right.$ and to $\left.X(w)=\left(\partial^{\gamma} \bar{f}(w)\right)_{|\gamma| \leq l\left(M^{\prime}\right)}\right)$ for $j=1, \ldots, n$, we obtain the following result.

Proposition 7.1. Let $M \subset \mathbb{C}^{n}$ be a real analytic generic submanifold through the origin and $M^{\prime} \subset \mathbb{C}^{n}$ be a real algebraic generic submanifold through the origin (with the same CR dimension). Let $f: M \rightarrow M^{\prime}$ be a formal nondegenerate $C R$ map between $M$ and $M^{\prime}$ and assume that $M^{\prime}$ is holomorphically nondegenerate. Then, for any multi-index $\mu \in \mathbb{N}^{n}$ and for any $j \in\{1, \ldots, n\}$, there exists a positive integer $l(\mu, j)$, a family of convergent power series $\delta_{i \mu j}=\delta_{i \mu j}\left(\left(\Lambda_{\gamma}\right)_{|\gamma| \leq l\left(M^{\prime}\right)+|\mu|}, z, w\right) \in \mathbb{C}\left\{\left(\Lambda_{\gamma}-\right.\right.$ $\left.\left.\partial^{\gamma} \bar{f}(0)\right)_{|\gamma| \leq l\left(M^{\prime}\right)+|\mu|}, z, w\right\}, i=0, \ldots, l(\mu, j)$, such that the formal identity

$$
\sum_{i=0}^{l(\mu, j)} \delta_{i \mu j}\left(\left(\partial^{\gamma} \bar{f}(w)\right)_{|\gamma| \leq l\left(M^{\prime}\right)+|\mu|}, z, w\right)\left(\partial^{\mu} f_{j}(z)\right)^{i}=0,
$$

holds for $(z, w) \in \mathcal{M}$ with $\delta_{l(\mu, j) \mu j}\left(\left(\partial^{\gamma} \bar{f}(w)\right)_{|\gamma| \leq l\left(M^{\prime}\right)+|\mu|}, z, w\right) \neq 0$ on $\mathcal{M}$. 
If furthermore $M$ is assumed to be minimal at 0 , then, in view of (7.1), we may apply Proposition 5.3 to $h(z)=f_{j}(z)$ and $X(w)=\left(\partial^{\gamma} \bar{f}(w)\right)_{|\gamma| \leq l\left(M^{\prime}\right)}$, for $j=1, \ldots, n$. This gives the following proposition.

Proposition 7.2. Let $M \subset \mathbb{C}^{n}$ be a real analytic generic submanifold through the origin and $M^{\prime} \subset \mathbb{C}^{n}$ be a real algebraic generic submanifold through the origin. Let $f: M \rightarrow M^{\prime}$ be a formal nondegenerate CR map between $M$ and $M^{\prime}$ and assume that $M$ is minimal at 0 and that $M^{\prime}$ is holomorphically nondegenerate. Let $N$ be the $C R$ dimension of $M$ (and of $M^{\prime}$ ) and $v_{j}, j \in \mathbb{N}$, be the Segre sets mappings for $M$ as defined by (3.5) and (3.6). Then, for any multi-index $\mu \in \mathbb{N}^{n}$, for any $d \in \mathbb{N}$ and for any $j \in\{1, \ldots, n\}$, there exist two positive integers $l=l(\mu, d, j), p=p(\mu, d, j)$, and a family of convergent power series $\psi_{\nu j}^{\mu d}=\psi_{\nu j}^{\mu d}\left(\left(\Lambda_{\gamma}\right)_{|\gamma| \leq p}, z, w\right) \in \mathbb{C}\left\{\left(\Lambda_{\gamma}-\partial^{\gamma} \bar{f}(0)\right)_{|\gamma| \leq p}, z, w\right\}, \nu=0, \ldots, l$, such that the formal identity

$$
\sum_{\nu=0}^{l} \psi_{\nu j}^{\mu d}\left(\left(\left(\partial^{\gamma} \bar{f}\right) \circ \bar{v}_{d}\right)_{|\gamma| \leq p}, \bar{v}_{d}, v_{d+1}\right)\left(\left(\partial^{\mu} f_{j}\right) \circ v_{d+1}\right)^{\nu} \equiv 0
$$

holds in the ring of formal power series in $(d+1) N$ determinates and such that $\psi_{l j}^{\mu d}\left(\left(\left(\partial^{\gamma} \bar{f}\right) \circ \bar{v}_{d}\right)_{|\gamma| \leq p}, \bar{v}_{d}, v_{d+1}\right) \not \equiv 0$.

From Proposition 7.2, one sees that the convergence of the mapping $f$ (under the assumptions of Theorem 2.2) follows from successive applications of Lemma 5.7. Indeed, for $d=0$, Proposition 7.2, and Lemma 5.7 yield the convergence of $f$ and of all its jets on the first Segre set. From this, Proposition 7.2 and Lemma 5.7, we obtain the convergence of $f$ and of all its jets on the second Segre set, and so on. This leads to the convergence of $f$ on the $d_{0}$-th Segre set, where $d_{0}$ is given by Theorem 3.1. As in the proof of Theorem 5.1, this implies the convergence of $f$ under the assumptions of Theorem 2.2 .

\section{Partial convergence of formal CR maps.}

In this section, as in [21], we indicate several results which show how Theorem 2.1 can be seen as a result of partial convergence for formal nondegenerate CR maps. Before explaining what we mean by this, we have to recall several facts.

Let $M$ be a real analytic generic submanifold in $\mathbb{C}^{n}$ and $p \in M$. Let $\mathbb{K}(p)$ be the quotient field of $\mathbb{C}\{z-p\}$, and $H(M, p)$ be the vector space over 
$\mathbb{K}(p)$ consisting of the germs at $p$ of $(1,0)$ vector fields, with meromorphic coefficients, tangent to $M$ (near $p$ ). The degeneracy of $M$ at $p$, denoted $d(M, p)$, is defined to be the dimension of $H(M, p)$ over $\mathbb{K}(p)$. It is shown in $[8,3]$ that the mapping $M \ni p \mapsto d(M, p) \in\{0, \ldots, n\}$ is constant on any connected component of $M$. Consequently, if $M$ is a connected real analytic generic submanifold, one can define its degeneracy $d(M)$ to be the degeneracy $d(M, q)$ at any point $q \in M$. Observe that the germ $(M, p)$, $p \in M$, is holomorphically nondegenerate if and only if $d(M)=d(M, p)=0$.

If $f$ is a formal nondegenerate CR map as in Theorem 2.1, $f$ can or cannot be convergent. The following result, which is of more interest when $f$ is not convergent, shows however that the map $f$ is partially convergent in the following sense.

Theorem 8.1. Let $f:(M, 0) \rightarrow\left(M^{\prime}, 0\right)$ be a formal nondegenerate $C R$ map between two germs at 0 in $\mathbb{C}^{n}$ of real analytic generic submanifolds. Assume that $M$ is minimal at 0 and that $M^{\prime}$ is real algebraic. Let $d\left(M^{\prime}\right)$ be the degeneracy of $M^{\prime}$. Then there exists a holomorphic (algebraic) mapping (independent of $f) \mathcal{G}(\omega)=\left(\mathcal{G}_{1}(\omega), \ldots, \mathcal{G}_{n-d\left(M^{\prime}\right)}(\omega)\right)$ defined near $0 \in \mathbb{C}^{n}$ of generic rank $n-d\left(M^{\prime}\right)$ such that $\mathcal{G} \circ f$ is convergent.

Proof. We use again the notations of $\S 2$ and $\S 4.1$. As in the proof of Theorem 2.2 , by using the expansion (4.3), we obtain for $\nu=1, \ldots, c$,

$$
\bar{\Phi}_{\nu}^{\prime}(f(z), \theta)=\sum_{\beta \in \mathbb{N}^{N}}\left(q_{\beta, \nu} \circ f\right)(z) \theta^{\beta} .
$$

Recall also that the $q_{\beta, \nu}(\omega)$ are algebraic functions. By Theorem 2.1, we have that for any $\beta \in \mathbb{N}^{N}$ and for any $\nu=1, \ldots, c, q_{\beta, \nu} \circ f$ is convergent in some neighborhood of 0 in $\mathbb{C}^{n}$. According to $[8,3]$, we can choose $q_{\beta^{1}, \nu^{1}}(\omega), \ldots, q_{\beta^{r}, \nu^{r}}(\omega), r=n-d\left(M^{\prime}\right)$, of generic rank $n-d\left(M^{\prime}\right)$ in a neighborhood of 0 in $\mathbb{C}^{n}$. Then, if we define $\mathcal{G}_{i}(\omega)=q_{\beta^{i}, \nu^{i}}(\omega)$, for $i=1, \ldots, n-d\left(M^{\prime}\right)$, we obtain the desired statement of the Theorem.

A suitable invariant which also measures the lack of convergence of a given formal (holomorphic) mapping is its so-called transcendence degree. We recall first how such an invariant is defined (cf. [21]). Let $\mathcal{H}:\left(\mathbb{C}^{N}, 0\right) \rightarrow$ $\left(\mathbb{C}^{N^{\prime}}, 0\right)$ be a formal holomorphic mapping, $N, N^{\prime} \geq 1$, and $V$ be a complex analytic set through the origin in $\mathbb{C}^{N} \times \mathbb{C}^{N^{\prime}}$. Assume that $V$ is given near the origin in $\mathbb{C}^{N+N^{\prime}}$ by $V=\left\{(x, y) \in \mathbb{C}^{N} \times \mathbb{C}^{N^{\prime}}: \varrho_{1}(x, y)=\ldots=\varrho_{q}(x, y)=0\right\}$, $\varrho_{i}(x, y) \in \mathbb{C}\{x, y\}, i=1, \ldots, q$. Then, the graph of $\mathcal{H}$ is said to be formally 
contained in $V$ if $\varrho_{1}(x, \mathcal{H}(x))=\ldots=\varrho_{q}(x, \mathcal{H}(x))=0$ in $\mathbb{C}[[x]]$. Furthermore, if $V_{\mathcal{H}}$ is the germ of the complex analytic set through the origin in $\mathbb{C}^{N+N^{\prime}}$ defined as the intersection of all complex analytic sets through the origin in $\mathbb{C}^{N+N^{\prime}}$ formally containing the graph of $\mathcal{H}$, then the transcendence degree of $\mathcal{H}$ is defined to be the integer $\operatorname{dim}_{\mathbb{C}} V_{\mathcal{H}}-N$. This definition is in complete analogy with the one introduced in [10] in the $\mathcal{C}^{\infty}$ mapping problem. The following proposition from [21] shows the relevance of the previous concept and why this transcendence degree is related to the convergence properties of formal mappings.

Proposition 8.2. Let $\mathcal{H}:\left(\mathbb{C}^{N}, 0\right) \rightarrow\left(\mathbb{C}^{N^{\prime}}, 0\right)$ be formal holomorphic mapping. Then the following conditions are equivalent:

i) $\mathcal{H}$ is convergent;

ii) The transcendence degree of $\mathcal{H}$ is zero.

The following is a consequence of Theorem 8.1.

Corollary 8.3. Let $f:(M, 0) \rightarrow\left(M^{\prime}, 0\right)$ be a formal $C R$ mapping between two germs at 0 of real analytic generic submanifolds. Assume that $M$ is minimal at $0, M^{\prime}$ is real algebraic and that $f$ is nondegenerate, i.e., $J_{f} \not \equiv 0$. Denote by $\mathcal{D}_{f}$ the transcendence degree of $f$. Then $\mathcal{D}_{f} \leq d\left(M^{\prime}\right)$, where $d\left(M^{\prime}\right)$ denotes the degeneracy of $M^{\prime}$. Equivalently, there exists a complex analytic set of (pure) dimension $n+d\left(M^{\prime}\right)$ which contains formally the graph of $f$.

Proof. The proof is similar to the one given in [21]. For the sake of completeness, we recall it. From Theorem 8.1, there exists $\mathcal{G}(\omega)=$ $\left(\mathcal{G}_{1}(\omega), \ldots, \mathcal{G}_{n-d\left(M^{\prime}\right)}(\omega)\right) \in(\mathbb{C}\{\omega\})^{n-d\left(M^{\prime}\right)}$ of generic rank $n-d\left(M^{\prime}\right)$ such that $\mathcal{G} \circ f$ is convergent. Put $\delta_{j}(z):=\left(\mathcal{G}_{j} \circ f\right)(z) \in \mathbb{C}\{z\}$, for $j=$ $1, \ldots, n-d\left(M^{\prime}\right)$. Then, the graph of $f$ is formally contained in the complex analytic set

$$
A=\left\{(z, \omega) \in\left(\mathbb{C}^{2 n}, 0\right): \mathcal{G}_{j}(\omega)=\delta_{j}(z), j=1, \ldots, n-d\left(M^{\prime}\right)\right\} .
$$

Let $A=\cup_{i=1}^{k} \Gamma_{i}$ be the decomposition of $A$ into irreducible components. For any positive integer $\sigma$, one can find, according to the Artin approximation theorem [1], a convergent power series mapping $f^{\sigma}(z) \in(\mathbb{C}\{z\})^{n}$ defined in some small neighborhood $U^{\sigma}$ of 0 in $\mathbb{C}^{n}$, which agrees with $f(z)$ up to order $\sigma$ (at 0 ) and such that the graph of $f^{\sigma}$, denoted $G\left(f^{\sigma}\right)$, is contained 
in $A$. Since $G\left(f^{\sigma}\right)$ is contained in $A$, it must be contained in an irreducible component of $A$. Thus, at least one subsequence of $\left(f^{\sigma}\right)_{\sigma \in \mathbb{N}^{*}}$ is contained in one of such irreducible components, say $\Gamma_{1}$. We can assume without loss of generality that such a subsequence is $\left(f^{\sigma}\right)_{\sigma \in \mathbb{N}^{*}}$ itself. We first observe that this implies that the graph of $f$ is formally contained in $\Gamma_{1}$. Moreover, since $f$ is a formal nondegenerate map, for $\sigma_{0}$ large enough, the family $\left(f^{\sigma}\right)_{\sigma \geq \sigma_{0}}$ is also a family of holomorphic maps of generic rank $n$. In particular, this implies that the generic rank of the family of holomorphic functions

$$
\left(\left(\mathcal{G}_{i} \circ f^{\sigma_{0}}\right)(z)\right)_{1 \leq i \leq n-d\left(M^{\prime}\right)},
$$

is $n-d\left(M^{\prime}\right)$. As a consequence, if $z_{0}$ is close enough to 0 in $\mathbb{C}^{n}$ and is chosen so that the rank of the preceding family at $z_{0}$ equals $n-d\left(M^{\prime}\right)$, the implicit function theorem shows that $A$ is an $n+d\left(M^{\prime}\right)$-dimensional complex submanifold near $\left(z_{0}, f^{\sigma_{0}}\left(z_{0}\right)\right) \in \Gamma_{1}$. Since $\Gamma_{1}$ is irreducible, it is puredimensional, and thus, $\Gamma_{1}$ is an $n+d\left(M^{\prime}\right)$ pure-dimensional complex analytic set containing formally the graph of $f$. By definition of the transcendence degree, this implies that $\mathcal{D}_{f} \leq d\left(M^{\prime}\right)$.

Remark 8. One should observe that Theorem 2.2 also follows from Corollary 8.3. Indeed, if, in Corollary 8.3, $M^{\prime}$ is furthermore assumed to be holomorphically nondegenerate, then $d\left(M^{\prime}\right)=0$ and thus the transcendence degree of $f$ is zero. By Proposition 8.2, this implies that $f$ is convergent.

\section{Concluding remarks.}

In this last section, we indicate how Theorem 5.1 can be applied to the study of the convergence of formal mappings between real analytic CR manifolds in complex spaces of possibly different dimensions. Our last result will be expressed by means of a standard nondegeneracy condition which takes its source in $[23,26,12]$. The situation is the following one.

Let $f:(M, 0) \rightarrow\left(M^{\prime}, 0\right)$ be a formal CR mapping between two germs at 0 of real analytic generic submanifolds in $\mathbb{C}^{n}$ and $\mathbb{C}^{n^{\prime}}$ respectively, $n, n^{\prime} \geq 2$. (We wish to mention that all the following considerations are also valid for a target real analytic set, but for simplicity, we restrict our attention to generic manifolds.) We shall use the notations defined in $\S 3$ for $M$. In particular, the CR dimension of $M$ is $N$ and its real codimension is $c$. Following the terminology of [10], we define the characteristic variety of $f$ at $0 \in \mathbb{C}^{n^{\prime}}$ as follows. If $M^{\prime}$ is a real analytic generic submanifold through 0 as above, of CR dimension $N^{\prime}$ and of real codimension $c^{\prime}$, we can assume that it is given 
near 0 by

$$
M^{\prime}=\left\{\zeta \in\left(\mathbb{C}^{n^{\prime}}, 0\right): \rho^{\prime}(\zeta, \bar{\zeta})=0\right\}
$$

with

$$
\bar{\partial} \rho_{1}^{\prime} \wedge \ldots \wedge \bar{\partial} \rho_{c^{\prime}}^{\prime} \neq 0, \text { on } M^{\prime}
$$

Here, $\rho^{\prime}=\left(\rho_{1}^{\prime}, \ldots, \rho_{c^{\prime}}^{\prime}\right)$ is a set of local real analytic defining functions for $M^{\prime}$ near $0 \in \mathbb{C}^{n^{\prime}}$. Consider the vector fields $\mathcal{L}_{j}, j=1, \ldots, N$, tangent to the complexification $\mathcal{M}$ of the source manifold $M$ as defined by (3.3). It will be better to see these vector fields, for fixed $z \in\left(\mathbb{C}^{n}, 0\right)$, as a basis of holomorphic vector fields tangent to the Segre variety $Q_{\bar{z}}$, and thus, we shall denote them $\mathcal{L}_{(z, w)}^{j}$ for $j=1, \ldots, N$. For any multi-index $\gamma=\left(\gamma_{1}, \ldots, \gamma_{N}\right) \in$ $\mathbb{N}^{N}$, we define $\mathcal{L}_{(z, w)}^{\gamma}=\left[\mathcal{L}_{(z, w)}^{1}\right]^{\gamma_{1}} \ldots\left[\mathcal{L}_{(z, w)}^{N}\right]^{\gamma_{N}}$. Finally, for any multi-index $\gamma \in \mathbb{N}^{N}$, let $\Xi_{\gamma}$ be the $\mathbb{C}^{c^{\prime}}$ formal map defined by

$$
\mathbb{C}^{n} \times \mathbb{C}^{n} \times \mathbb{C}^{n^{\prime}} \ni(z, w, \zeta) \mapsto \mathcal{L}_{(z, w)}^{\gamma} \rho^{\prime}(\zeta, \bar{f}(w)) \in \mathbb{C}^{c^{\prime}} .
$$

Observe that there exists a $\mathbb{C}^{c^{\prime}}$-valued convergent power series mapping $\left.\mathcal{N}_{\gamma}=\mathcal{N}_{\gamma}\left(\left(\Lambda_{\beta}\right)_{|\beta| \leq|\gamma|}, z, w, \zeta\right) \in\left(\mathbb{C}\left\{\Lambda_{0}, z, w, \zeta\right\}\left[\left(\Lambda_{\beta}\right)\right)_{1 \leq|\beta| \leq|\gamma|}\right]\right)^{c^{\prime}}$ such that

$$
\Xi_{\gamma}(z, w, \zeta)=\mathcal{N}_{\gamma}\left(\left(\partial^{\beta} \bar{f}(w)\right)_{|\beta| \leq|\gamma|}, z, w, \zeta\right) \text {, in } \mathbb{C}[[z, w, \zeta]] .
$$

The characteristic variety of $f$ at $0 \in \mathbb{C}^{n}$ is then defined to be the germ at $0 \in \mathbb{C}^{n^{\prime}}$ of the complex analytic set

$$
\mathcal{C}(f, 0)=\left\{\zeta \in\left(\mathbb{C}^{n^{\prime}}, 0\right): \Xi_{\gamma}(0,0, \zeta)=0, \gamma \in \mathbb{N}^{N}\right\} .
$$

This set is the infinitesimal analog of the usual determinacy set for holomorphic mappings

$$
\left\{\zeta \in\left(\mathbb{C}^{n^{\prime}}, 0\right): f\left(Q_{0}\right) \subset Q_{\zeta}^{\prime}\right\}
$$

where $Q_{\zeta}^{\prime}$ is the Segre variety associated to $M^{\prime}$ and $\zeta \in\left(\mathbb{C}^{n^{\prime}}, 0\right)$. With this in mind, we have the following natural result.

Theorem 9.1. Let $f:(M, 0) \rightarrow\left(M^{\prime}, 0\right)$ be a formal mapping between two germs at 0 of real analytic generic submanifolds in $\mathbb{C}^{n}$ and $\mathbb{C}^{n^{\prime}}$ respectively. Assume that $M$ is minimal at 0 and that the characteristic variety $\mathcal{C}(f, 0)$ is zero-dimensional. Then $f$ is convergent.

Proof. Since $f$ maps formally $M$ to $M^{\prime}$, we have

$$
\rho^{\prime}(f(z), \bar{f}(w))=0
$$


as a formal power series identity for $(z, w) \in \mathcal{M}$. Thus, if, for $\gamma \in \mathbb{N}^{N}$, we apply $\mathcal{L}_{(z, w)}^{\gamma}$ to $(9.3)$, it follows from the definition of $\Xi_{\gamma}$ given in (9.1), that

$$
\mathcal{L}_{(z, w)}^{\gamma}\left(\rho^{\prime}(f(z), \bar{f}(w))\right)=\Xi_{\gamma}(z, w, f(z))=0
$$

for $(z, w) \in \mathcal{M}$. Observe that it follows from (9.2) and (9.4) that

$$
\mathcal{N}_{\gamma}\left(\left(\partial^{\beta} \bar{f}(w)\right)_{|\beta| \leq|\gamma|}, z, w, f(z)\right)=0, \text { for }(z, w) \in \mathcal{M}
$$

Since the characteristic variety $\mathcal{C}(f, 0)$ is zero-dimensional, in view of (9.2), the holomorphic mapping $\mathcal{I}_{k}\left({ }^{2}\right)$

$$
\begin{aligned}
\mathbb{C}^{n^{\prime} d(n, k)+2 n+n^{\prime}} \ni( & \left.\left(\Lambda_{\beta}\right)_{|\beta| \leq k}, z, w, \zeta\right) \mapsto \\
& \left(\left(\Lambda_{\beta}\right)_{|\beta| \leq k}, z, w,\left(\mathcal{N}_{\gamma}\left(\left(\Lambda_{\beta}\right)_{|\beta| \leq|\gamma|}, z, w, \zeta\right)\right)_{|\gamma| \leq k}\right)
\end{aligned}
$$

is finite-to-one near $J_{0}=\left(\left(\partial^{\beta} \bar{f}(0)\right)_{|\beta| \leq k}, 0,0,0\right) \in \mathbb{C}^{n^{\prime}} d(n, k)+2 n+n^{\prime}$ for $k$ large enough. It then follows from [16] (p.15) (see also [18]) that, for any $j=$ $1, \ldots, n^{\prime}, \zeta_{j}$ is integral over the ring formed by all the convergent power series of the form

$$
\left(\mathcal{B} \circ \mathcal{I}_{k}\right)\left(\left(\Lambda_{\beta}\right)_{|\beta| \leq k}, z, w, \zeta\right)
$$

$\mathcal{B}$ running over all the convergent power series centered at

$$
J_{1}=\left(\left(\partial^{\beta} \bar{f}(0)\right)_{|\beta| \leq k}, 0,0,0\right) \in \mathbb{C}^{n^{\prime} d(n, k)+2 n+c^{\prime} d(N, k)} .
$$

Explicitly, for any $j=1, \ldots, n^{\prime}$, there exists a positive integer $\nu_{j}$ and convergent power series $\mathcal{B}_{t, j}$ near $J_{1}, t=0, \ldots, \nu_{j}-1$, such that the following identities hold in a neigborhood of $J_{0}$ :

$$
\zeta_{j}^{\nu_{j}}+\sum_{t<\nu_{j}}\left(\mathcal{B}_{t, j} \circ \mathcal{I}_{k}\right)\left(\left(\Lambda_{\beta}\right)_{|\beta| \leq k}, z, w, \zeta\right) \quad \zeta_{j}^{t}=0
$$

Putting $\zeta=f(z)$ and $\left(\Lambda_{\beta}\right)_{|\beta| \leq k}=\left(\partial^{\beta} \bar{f}(w)\right)_{|\beta| \leq k}$ for $(z, w) \in\left(\mathbb{C}^{2 n}, 0\right)$ in (9.7), we obtain that for $(z, w) \in\left(\mathbb{C}^{2 n}, 0\right)$ and for $j=1, \ldots, n^{\prime}$, the following formal identities hold in $\mathbb{C}[[z, w]]$ :

$$
\left(f_{j}(z)\right)^{\nu_{j}}+\sum_{t<\nu_{j}}\left(\mathcal{B}_{t, j} \circ \mathcal{I}_{k}\right)\left(\left(\partial^{\beta} \bar{f}(w)\right)_{|\beta| \leq k}, z, w, f(z)\right)\left(f_{j}(z)\right)^{t}=0 .
$$

\footnotetext{
${ }^{2} d(i, j)=\operatorname{Card}\left\{\alpha \in \mathbb{N}^{i}:|\beta| \leq j\right\}, i, j \in \mathbb{N}^{*}$.
} 
But in view of the definition of $\mathcal{I}_{k}$ given in (9.6) and in view of (9.5), we have for $j=1, \ldots, n^{\prime}$, for $t=0, \ldots, \nu_{j}-1$ and for $(z, w) \in \mathcal{M}$,

$$
\begin{aligned}
& \left(\mathcal{B}_{t, j} \circ \mathcal{I}_{k}\right)\left(\left(\partial^{\beta} \bar{f}(w)\right)_{|\beta| \leq k}, z, w, f(z)\right) \\
& =\mathcal{B}_{t, j}\left(\left(\partial^{\beta} \bar{f}(w)\right)_{|\beta| \leq k}, z, w,\left(\mathcal{N}_{\gamma}\left(\left(\partial^{\beta} \bar{f}(w)\right)_{|\beta| \leq|\gamma|}, z, w, f(z)\right)\right)_{|\gamma| \leq k}\right) \\
& =\mathcal{B}_{t, j}\left(\left(\partial^{\beta} \bar{f}(w)\right)_{|\beta| \leq k}, z, w, 0\right) .
\end{aligned}
$$

Thus, from (9.8), we obtain for $j=1, \ldots, n^{\prime}$,

$$
\left(f_{j}(z)\right)^{\nu_{j}}+\sum_{t<\nu_{j}} \mathcal{B}_{t, j}\left(\left(\partial^{\beta} \bar{f}(w)\right)_{|\beta| \leq k}, z, w, 0\right)\left(f_{j}(z)\right)^{t}=0
$$

on $\mathcal{M}$. As a consequence, we see that for each $j=1, \ldots, n^{\prime}$, the formal holomorphic power series $f_{j}(z), j=1, \ldots, n^{\prime}$, satisfies (ii) of Theorem 5.1. Since $M$ is minimal at 0 , from that theorem, we conclude that $f$ is convergent.

Remark 9. It should be mentioned that Theorem 9.1 above could also be derived from the techniques of [6].

We conclude by mentioning several situations where Theorem 9.1 applies. It contains the cases of formal invertible mappings of Levi-nondegenerate real analytic hypersurfaces, finite mappings of minimal essentially finite real analytic generic manifolds or, more generally, mappings with injective Segre homomorphism (in the sense of [6]) from minimal real analytic generic manifolds into real analytic essentially finite ones (the proof is contained in [6]).

\section{References.}

[1] M. Artin, On the solution of analytic equations, Invent. Math., 5 (1968), 277-291.

[2] M.S. Baouendi, P. Ebenfelt and L.P. Rothschild, Real Submanifolds in Complex Space and Their Mappings, Princeton University Press, N.J., 1999.

[3] M.S. Baouendi, P. Ebenfelt and L.P. Rothschild, Algebraicity of holomorphic mappings between real algebraic sets in $\mathbb{C}^{n}$, Acta Math., 177 (1996), 225-273. 
[4] M.S. Baouendi, P. Ebenfelt and L.P. Rothschild, Parametrization of local biholomorphisms of real analytic hypersurfaces, Asian J. Math., 1 (1997), 1-16.

[5] M.S. Baouendi, P. Ebenfelt and L.P. Rothschild, Rational dependence of smooth and analytic CR mappings on their jets, Math. Ann., 315 (1999), 205-249.

[6] M.S. Baouendi, P. Ebenfelt and L.P. Rothschild, Convergence and finite determination of formal CR mappings, J. Amer. Math. Soc., 13 (2000), 697-723.

[7] M.S. Baouendi, H. Jacobowitz and F. Treves, On the analyticity of $C R$ mappings, Ann. of Math., 122 (1985), 365-400.

[8] M.S. Baouendi and L.P. Rothschild, Mappings of real algebraic hypersurfaces, J. Amer. Math. Soc., 8 (1995), 997-1015.

[9] S.S. Chern and J.K. Moser, Real hypersurfaces in complex manifolds, Acta Math., 133 (1974), 219-271.

[10] B. Coupet, S. Pinchuk and A. Sukhov, Analyticité des applications CR, C. R. Acad. Sci. Paris, 329, Série I, (1999), 489-494.

[11] M. Derridj, Prolongement local d'applications holomorphes propres en des points faiblement pseudoconvexes, Prépublication Orsay 84T45, 1984.

[12] K. Diederich and J.E. Fornæss, Proper holomorphic mappings between real analytic pseudoconvex domains in $\mathbb{C}^{n}$, Math. Ann., 282 (1988), 681-700.

[13] K. Diederich and S. Pinchuk, Proper holomorphic maps in dimension two extend, Indiana Univ. Math. J., 44 (1995), 1089-1126.

[14] K. Diederich and S. Webster, A reflection principle for degenerate real hypersurfaces, Duke Math. J., 47 (1980), 835-843.

[15] X. Gong, Divergence of the normalization for real Lagrangian surfaces near complex tangents, Pac. J. Math., 176 (1996), 311-324.

[16] R. C. Gunning, Lectures on complex analytic varieties: Finite analytic mappings, Princeton University Mathematical Series, Princeton University Press, N. J., 1973. 
[17] X. Huang, Schwarz reflection principle in complex spaces of dimension two, Comm. Part. Diff. Eq., 21 (1996), 1781-1829.

[18] N. Mir, Analyticité et algébricité d'applications CR ou holomorphes, Thesis. Université de Rouen, France, Juin 1998.

[19] N. Mir, An algebraic characterization of holomorphic nondegeneracy for real algebraic hypersurfaces and its application to CR mappings, Math. Z., 231 (1999), 189-202.

[20] N. Mir, Germs of holomorphic mappings between real algebraic hypersurfaces, Ann. Inst. Fourier, Grenoble, 48 (1998), 1025-1043.

[21] N. Mir, Formal biholomorphic maps of real analytic hypersurfaces, Math. Res. Lett., 7 (2000), 343-359.

[22] J.K. Moser and S. Webster, Normal forms for real surfaces in $\mathbb{C}^{2}$ near complex tangents and hyperbolic surface transformations, Acta Math., 150 (1983), 255-296.

[23] S. Pinchuk, On the analytic continuation of holomorphic mappings, Math. USSR Sb., 27 (1975), 375-392.

[24] N. Stanton, Infinitesimal CR automorphisms of real hypersurfaces, Amer. J. Math, 118 (1996), 209-233.

[25] A.E. Tumanov, Extending CR functions into a wedge from a manifold of finite type, (English Translation) Math. USSR-Sb. 64, (1989), 129140.

[26] S. Webster, On the mapping problem for algebraic real hypersurfaces, Invent. Math., 43 (1977), 53-68.

[27] S. Webster, Holomorphic symplectic normalization of a real function, Ann. Sc. Norm. Sup. Pisa, 19 (1992), 69-86.

[28] O. Zariski and P. Samuel, Commutative algebra, Volumes 1, 2, Van Nostrand, 1958.

UNIVERSITÉ DE ROUEN

Laboratoire de Mathématiques Raphä̈l Salem

UMR 6085 CNRS, Site COLBERT

76821 Mont Saint Aignan, France

E-mail address: Nordine.Mir@univ-rouen.fr

Received September 29, 1999 and Revised February 3, 2000. 